\title{
SN 2017ivv: two years of evolution of a transitional Type II supernova
}

\author{
C. P. Gutiérrez ${ }^{\oplus, 1 \star}$ A. Pastorello, ${ }^{2}$ A. Jerkstrand, ${ }^{3,4}$ L. Galbany ${ }^{\odot},{ }^{5}$ M. Sullivan ${ }^{\odot},{ }^{1}$ J. P. Anderson, ${ }^{6}$ \\ S. Taubenberger, ${ }^{7}$ H. Kuncarayakti, ${ }^{8,9}$ S. González-Gaitán ${ }^{\odot},{ }^{10}$ P. Wiseman ${ }^{\odot},{ }^{1}$ C. Inserra ${ }^{\odot}, 11$ \\ M. Fraser ${ }^{\odot},{ }^{12}$ K. Maguire ${ }^{\odot},{ }^{13}$ S. Smartt, ${ }^{14}$ T. E. Müller-Bravo ${ }^{\oplus},{ }^{1}$ I. Arcavi, ${ }^{15,16}$ S. Benetti, ${ }^{17}$ D. Bersier, ${ }^{18}$ \\ S. Bose ${ }^{\oplus},{ }^{19,20}$ K. A. Bostroem, ${ }^{21}$ J. Burke, ${ }^{22,23}$ P. Chen, ${ }^{24,25}$ T.-W. Chen, ${ }^{7,26}$ M. Della Valle, ${ }^{27,28}$ \\ Subo Dong, ${ }^{24}$ A. Gal-Yam, ${ }^{29}$ M. Gromadzki, ${ }^{30}$ D. Hiramatsu, ${ }^{22,23}$ T. W.-S. Holoien ${ }^{\oplus},{ }^{31} \dagger$ \\ G. Hosseinzadeh, ${ }^{32}$ D. A. Howell, $, 22,23$ E. Kankare, ${ }^{8}$ C. S. Kochanek ${ }^{\oplus}, 19,20$ C. McCully, ${ }^{22,23}$ \\ M. Nicholl, ${ }^{33,34}$ G. Pignata, ${ }^{35,36}$ J. L. Prieto, ${ }^{36,37}$ B. Shappee, ${ }^{38}$ K. Taggart ${ }^{\oplus},{ }^{18}$ L. Tomasella ${ }^{\oplus},{ }^{17}$ \\ S. Valenti ${ }^{21}$ and D. R. Young ${ }^{14}$ \\ Affiliations are listed at the end of the paper
}

Accepted 2020 September 3. Received 2020 September 3; in original form 2020 May 7

\begin{abstract}
We present the photometric and spectroscopic evolution of the Type II supernova (SN II) SN 2017ivv (also known as ASASSN17qp). Located in an extremely faint galaxy ( $\left.M_{r}=-10.3 \mathrm{mag}\right)$, SN 2017ivv shows an unprecedented evolution during the $2 \mathrm{yr}$ of observations. At early times, the light curve shows a fast rise $(\sim 6-8 \mathrm{~d})$ to a peak of $M_{g}^{\max }=-17.84 \mathrm{mag}$, followed by a very rapid decline of $7.94 \pm 0.48 \mathrm{mag}$ per $100 \mathrm{~d}$ in the $V$ band. The extensive photometric coverage at late phases shows that the radioactive tail has two slopes, one steeper than that expected from the decay of ${ }^{56} \mathrm{Co}$ (between 100 and $350 \mathrm{~d}$ ), and another slower (after $450 \mathrm{~d}$ ), probably produced by an additional energy source. From the bolometric light curve, we estimated that the amount of ejected ${ }^{56} \mathrm{Ni}$ is $\sim 0.059 \pm 0.003 \mathrm{M} \odot$. The nebular spectra of SN 2017ivv show a remarkable transformation that allows the evolution to be split into three phases: (1) $\mathrm{H} \alpha$ strong phase (<200 d); (2) $\mathrm{H} \alpha$ weak phase (between 200 and 350 d); and (3) $\mathrm{H} \alpha$ broad phase ( $>500 \mathrm{~d}$ ). We find that the nebular analysis favours a binary progenitor and an asymmetric explosion. Finally, comparing the nebular spectra of SN 2017ivv to models suggests a progenitor with a zero-age main-sequence mass of $15-17 \mathrm{M}_{\odot}$.
\end{abstract}

Key words: techniques: photometric-techniques: spectroscopic-surveys-supernovae: general.

\section{INTRODUCTION}

Core collapse supernovae (CC-SNe) are produced by the explosion of massive stars $\left(>8 \mathrm{M}_{\odot}\right)$. Observationally, they are a heterogeneous class, showing a large diversity in both spectra and photometry. They are broadly separated into objects with hydrogen in the spectra (Type II SNe; SNe II), and objects with no evidence of hydrogen lines (SNe I; Minkowski 1941). These hydrogen-poor SNe are classified as Type $\mathrm{Ib}$ or Type $\mathrm{Ic} \mathrm{SNe}(\mathrm{SNe} \mathrm{Ib} / \mathrm{c})$ based on the presence or absence of helium lines, respectively (Filippenko 1997; Gal-Yam 2017; Modjaz, Gutiérrez \& Arcavi 2019).

Based on light-curve shapes, SNe II were initially subclassified into $\mathrm{SNe}$ with linear fast-declining light curves (SNe IIL), and $\mathrm{SNe}$ with a slow decline or a plateau (SNe IIP; Barbon, Ciatti \& Rosino 1979). However, this separation has been refined with the analysis of larger samples of events that show a continuum in their photometric (e.g. Anderson et al. 2014b; González-Gaitán et al. 2015; Sanders et al. 2015; Rubin et al. 2016; Valenti et al. 2016; Galbany et al. 2016a) and spectroscopic properties (e.g. Gutiérrez

^ E-mail: C.P.Gutierrez-Avendano@ soton.ac.uk

$\dagger$ Carnegie Fellow et al. 2014, 2017b), suggesting that all these events come from a similar progenitor population.

Transitional objects between $\mathrm{SNe}$ II and $\mathrm{SNe} \mathrm{Ib}$ were identified with the discovery of SN 1987K (Filippenko 1988). This new class, identified as SNe IIb (Filippenko, Matheson \& Ho 1993), encompasses objects showing broad hydrogen features in their spectra at early epochs consistent with an SN II, but showing prominent helium lines at later phases, like $\mathrm{SNe} \mathrm{Ib}$. This transitional class is thought to arise from stars that have lost most, but not all, of their hydrogen envelopes prior to explosion. How this happens is not fully understood, but it may occur via binary interaction (e.g. Podsiadlowski, Joss \& Hsu 1992) or mass-loss through stellar winds (e.g. Heger et al. 2003; Puls, Vink \& Najarro 2008).

The direct identification of nearby $\mathrm{CC}-\mathrm{SN}$ progenitors in preexplosion images has revealed that $\mathrm{SNe}$ IIP arise from red supergiant (RSG) stars with masses of $8-18 \mathrm{M}_{\odot}$ (e.g. Van Dyk, Li \& Filippenko 2003; Smartt et al. 2004, 2009; Smartt 2015), while fast-declining objects (SN IIL) may arise from more massive progenitors or binary systems (Elias-Rosa et al. 2010, but see Maund et al. 2015). The direct detection of the progenitor of SN 1993J (e.g. Van Dyk et al. 2002; Maund et al. 2004; Maund \& Smartt 2009) and SN 2011dh (e.g. Maund et al. 2011; Van Dyk et al. 2011) suggests that many $\mathrm{SNe}$ IIb arise from stars in binary systems (see also Bersten et al. 2012; Benvenuto, Bersten \& Nomoto 2013). 
Within the single progenitor star scenario, a continuum between SNe IIL and IIb is predicted (Nomoto et al. 1996; Heger et al. 2003; Bayless et al. 2015); however, observational studies show some disagreements. Arcavi et al. (2012) analysed the $R$-band light-curve shape of $15 \mathrm{SNe}$ II and found a subdivision into three classes: IIP, IIL, and IIb, which were interpreted as different progenitor populations. Later on, Faran et al. (2014), analysing a sample of fast-declining SNe II, found that SNe IIL seem to be photometrically related to SNe IIb. More recently, Pessi et al. (2019), using a larger sample of $\mathrm{SNe}$ (73 SNe II and $22 \mathrm{SNe}$ IIb), found a lack of events that bridge the observed properties of these two classes, concluding that they form two observationally distinct families.

Theoretical work (e.g. Heger et al. 2003) has shown that metallicity plays an important role in the evolution and final fate of massive stars. At low metallicity, the mass-loss in massive stars is diminished (e.g. Woosley, Heger \& Weaver 2002) and the fraction of SNe IIP is higher (e.g. Heger et al. 2003; Woosley \& Heger 2015; Sravan, Marchant \& Kalogera 2019). At increased metallicity, mass-loss reduces the hydrogen envelope and only SNe IIL/IIb result (Heger et al. 2003). However, for single RSG stars, some studies (e.g. van Loon et al. 2005; Goldman et al. 2017; Chun et al. 2018) suggest that the massloss is independent of metallicity. If this is the case, the rate of RSG SN IIb progenitors at low and high metallicity must be similar. However, recent results from SN host studies (Galbany et al. 2018) show that $\mathrm{SNe}$ IIb environments are the most different from other CC-SNe: they occur in metal poor, and relatively low star formation rate environments (see also Arcavi et al. 2010). Galbany et al. (2018) argue that this result favours the binary system channel for SNe IIb.

While direct detections provide a relatively straightforward way to constrain the mass of the progenitor star, spectral modelling at late time ( $>100-150 \mathrm{~d}$ after explosion) can constrain the nucleosynthesis yields and thus the zero-age main-sequence (ZAMS) mass of the progenitor (Jerkstrand et al. 2012, 2014, 2015b, hereafter J15b). During late phases, the SN ejecta becomes optically thin in the continuum and the stellar layers corresponding to the helium core are exposed. Stellar evolution models (e.g. Woosley \& Weaver 1995) predict that the metal mass, particularly oxygen, strongly increases with progenitor ZAMS mass.

Although spectral modelling provides insights into the properties of the progenitor, to date, only a few nebular phase statistical studies of SNe II (Maguire et al. 2012; Jerkstrand et al. 2015a; Valenti et al. 2016; Silverman et al. 2017) and SNe IIb (Ergon et al. 2015, J15b, Fang \& Maeda 2018) have been published. Individual analyses of SNe IIb during the nebular phase (e.g. Matheson et al. 2000; Taubenberger et al. 2011) have shown a large diversity in this subclass, although a statistical characterization is still missing.

$\mathrm{SNe} \mathrm{IIb}$ are relatively rare events, representing $10-12$ percent of all CC-SNe ( $\mathrm{Li}$ et al. 2011; Shivvers et al. 2017), and only a few objects have been observed and analysed in detail. From these individual studies, SNe IIb have shown a large variety in their photometric properties that allows us to divide them into two groups: SNe IIb showing double-peaked light curves (e.g. SN 1993J, Richmond et al. 1994; Barbon et al. 1995; Matheson et al. 2000; SN 2011fu, Kumar et al. 2013; Morales-Garoffolo et al. 2015; SN 2016gkg Arcavi et al. 2017; Bersten et al. 2018) and SNe IIb with a single peak (e.g. SN 2008ax, Pastorello et al. 2008; SN 2011dh, Arcavi et al. 2011; Taubenberger et al. 2011; Sahu, Anupama \& Chakradhari 2013; Ergon et al. 2014, 2015; SN 2015as, Gangopadhyay et al. 2018). For the double-peak light curves, the initial peak, lasting less than $2 \mathrm{~d}$, has been attributed to the energy radiated by the shock (e.g. Woosley et al. 1994; Bersten et al. 2012; Nakar \& Piro 2014), while the second peak, with a duration of about
2 weeks, is powered by the decay of ${ }^{56} \mathrm{Ni}$. The double-peaked group is thought to arise from extended massive stars that interact with the dense wind from the progenitor, while the single-peaked group arises from compact stars (Chevalier \& Soderberg 2010).

In this paper, we present the analysis and results from the photometric and spectroscopic monitoring of SN 2017ivv over $2 \mathrm{yr}$. SN 2017ivv exploded in the faintest host galaxy reported for an SN II to date. Due to its exceptional behaviour, SN 2017ivv provides a great opportunity to explore and understand the connection and diversity within the SNe II and IIb classes, and the possible effects of metallicity in the $\mathrm{SN}$ evolution.

The paper is organized as follows. A description of the observations and data reduction are presented in Section 2. The host galaxy is characterized in Section 3. We describe the photometric and spectroscopic properties of SN 2017ivv in Sections 4 and 5, respectively. Finally, in Section 6, we present the analysis and discussion. We provide our conclusions in Section 7.

Throughout, we assume a flat $\Lambda \mathrm{CDM}$ universe, with a Hubble constant of $H_{0}=70 \mathrm{~km} \mathrm{~s}^{-1} \mathrm{Mpc}^{-1}$, and $\Omega_{\mathrm{m}}=0.3$.

\section{OBSERVATIONS OF SN 2017IVV}

\subsection{Discovery, explosion epoch, and classification}

SN 2017ivv (also known as ASASSN-17qp and ATLAS17ntk) was discovered by the All-Sky Automated Survey for Supernovae, ${ }^{1}$ ASAS-SN (Shappee et al. 2014; Kochanek et al. 2017) on 2017 December 12 (MJD = 58099.03; Brimacombe et al. 2017) with a magnitude of $g \sim 14.5$ mag. It was spectroscopically classified as an SN II by the Asiago Transient Classification Program (Tomasella et al. 2014) on 2017 December 15 (MJD = 58102.73). The last nondetection was obtained on 2017 December 4 (MJD = 58091.03), with a detection limit of $g \sim 17.14 \mathrm{mag}$. An early detection in the orange filter $(o=15.26 \mathrm{mag})$ was obtained on 2017 December 5 $(\mathrm{MJD}=58092.22)$ by the Asteroid Terrestrial-impact Last Alert System (ATLAS; Tonry et al. 2018; Smith et al. 2020). Given the first detection from ATLAS and last non-detection from ASASSN, we adopt 2017 December $4(\mathrm{MJD}=58091.62 \pm 0.59)$ as the explosion date.

SN 2017ivv was classified as a SN II based on the presence of hydrogen lines in the spectra. As part of the extended Public ESO Spectroscopic Survey of Transient Objects [ePESSTO; Smartt et al. (2015)], we started a follow-up campaign through the program 'SNe II in low-luminosity host galaxies'. To check the initial classification, the Supernova Identification (SNID, Blondin \& Tonry 2007) code and the GEneric cLAssification TOol (GELATO; Harutyunyan et al. 2008) were used and good matches for SN 2017ivv were found with several SNe II (e.g. SN 2007od, Inserra et al. 2011; SN 1995ad, Inserra et al. 2013) and a few SNe IIb (e.g. SN 2011fu, Kumar et al. 2013; Morales-Garoffolo et al. 2015). A H $\alpha$ P-Cygni profile with a broad emission component, but a weak absorption component suggested it is a fast-declining SN II. However, a gap in the observations between 20 and $100 \mathrm{~d}$ does not allow the study of the appearance of spectral lines and light-curve evolution during the recombination phase. When the $\mathrm{SN}$ became visible again, at $111 \mathrm{~d}$ from explosion, the spectra and light curve are still similar to fastdeclining SNe II. An analysis of the emission lines in the nebular spectra after $200 \mathrm{~d}$ suggest SN 2017ivv is not a typical hydrogen-rich $\mathrm{SN}$. It seems to have a lower hydrogen mass than any other object of

\footnotetext{
${ }^{1}$ http://www.astronomy.ohio-state.edu/asassn/index.shtml
} 
this type. As $\mathrm{SNe} \mathrm{IIb}$ are assumed to have much less hydrogen, we compare SN 2017ivv with both fast-declining SNe II and SNe IIb.

\subsection{Optical photometry}

Optical imaging of SN 2017ivv was acquired with several telescopes and instruments (Tables A1-A7, online supplementary material). Details of the observation are briefly summarized here:

(i) ATLAS: Photometry in the orange (o) filter (a red filter that covers $r+i$ ) was obtained by the twin 0.5-m ATLAS telescope system (Tonry et al. 2018), from 2017 December 5 to 2018 October 15. The ATLAS data were reduced and calibrated automatically as described in Tonry et al. (2018) and Smith et al. (2020). The mean magnitudes are reported in Table A1.

(ii) Las Cumbres Observatory: Most of the multiband optical photometry was obtained with the 1.0-m telescopes of Las Cumbres Observatory (Brown et al. 2013), through the ePESSTO and the Global Supernova Project allocated time. The photometric monitoring began on 2018 March 16, 3 months after the discovery and as soon as the $\mathrm{SN}$ was visible after being sun-constrained. An early observation in the BVri-bands was obtained on 2017 December 14. All data reduction was performed following the prescriptions described by Firth et al. (2015). The BgVri magnitudes of SN 2017ivv are presented in Table A2.

(iii) Asiago: Six epochs of $u B g$ Vriz photometry were obtained with the Copernico $1.82 \mathrm{~m}$ telescope equipped with AFOSF and the 67/91 Schmidt Telescope at the Asiago Observatory. The images were reduced following standard procedures (including bias, dark, and flat-field corrections). The ugriz magnitudes were calibrated using observations of local Sloan and Pan-STARRS sequences. The $B V$ magnitudes were derived using Pan-STARRS and the transformations in Chonis \& Gaskell (2008). Table A3 presents the Asiago photometry.

(iv) ASAS-SN: $V$ - and $g$-band photometric observations were obtained with the ASAS-SN units 'Brutus' (Hawaii), 'Cassius' (CTIO, Chile), and 'Henrietta Leavitt' (McDonald, Texas). All images were processed with an automated pipeline (Shappee et al. 2014), using the ISIS image subtraction package (Alard \& Lupton 1998; Alard 2000). Details of the process are presented in Shappee et al. (2014). We performed aperture photometry on the subtracted images using the IRAF APPHOT package, and then calibrated the results using the AAVSO Photometric All-Sky Survey (APASS; Henden et al. 2015). The ASAS-SN photometry is presented in Table A4.

(v) Post Observatory SRO: Five epochs of BVri photometry were acquired with the Apogee Alta U230 camera and the Apogee Alta U47 with $0.6 \mathrm{~m}$ telescopes at Post Observatory SRO (CA, USA) and Mayhill (NM, USA), respectively. All images were processed following standard procedures. Table A5 presents the Post photometry.

(vi) GROND: Two epochs of $r$-band and five epochs of $J H K$ photometry were obtained with the Gamma-Ray Burst Optical/NearInfrared Detector (GROND; Greiner et al. 2008) on the 2.2-m MPG telescope at the European Southern Observatory (ESO) La Silla Observatory in Chile. The images were reduced with the GROND pipeline (Krühler et al. 2008). The $r$ magnitudes were calibrated using Pan-STARRS, while the $J H K$ magnitudes were calibrated using 2MASS. The photometry is presented in Table A6.

(vii) MUSE: Two epochs of synthetic Vri-band photometry were extracted from the spectrum of SN 2017ivv obtained by the Multi Unit Spectroscopic Explorer (MUSE; Bacon et al. 2014) at the ESO Very Large Telescope (VLT). The MUSE data were reduced, using the ESOREFLEX pipeline (Freudling et al. 2013). Table A7 presents the MUSE synthetic photometry.

\subsection{Optical spectra}

Spectroscopic observations of SN 2017ivv were acquired with six different instruments: the ESO Faint Object Spectrograph and Camera (EFOSC2; Buzzoni et al. 1984) at the 3.5-m ESO New Technology Telescope (NTT), the Asiago Faint Object Spectrograph and Camera (AFOSC) at the 1.82-m Copernico Telescope, the Boller and Chivens spectrograph (BC) at the 1.22-m Galileo Telescope, the FOcal Reducer/low dispersion Spectrograph 2 (FORS2; Appenzeller et al. 1998), MUSE at the VLT, and the Low Resolution Imaging Spectrometer (LRIS; Oke et al. 1995) at the 10-m Keck telescope. In total, we obtained 25 epochs spanning between 10 and $671 \mathrm{~d}$ from explosion. Details of the instruments used for the spectral observations are reported in Table A8 (online supplementary material).

Data reductions for AFOSC and $\mathrm{BC}$ were performed with IRAF using standard routines (bias subtraction, flat-field correction, 1D extraction, and wavelength and flux calibration). For EFOSC2, the data were reduced using the PESSTO pipeline (Smartt et al. 2015). The spectrum obtained with FORS2 and the cube observed with MUSE were reduced using the ESOREFLEX pipeline (Freudling et al. 2013). The Keck LRIS spectrum was reduced with the LPIPE (Perley 2019) pipeline using default parameters and the standard reduction procedures. Finally, we calibrated the absolute flux of the spectra by using the $r$-band magnitudes to match the photometry. All spectra are available through the WISeREP ${ }^{2}$ archive (Yaron \& Gal-Yam 2012).

\section{THE HOST GALAXY}

The host galaxy of SN 2017ivv is GALEXASC J202849.46042255.5. It is a small and very faint galaxy with no previously published redshift. The only available information comes from a detection in Pan-STARRS 3Pi at a Kron mag of $g=21.27 \pm 0.06$ mag (Flewelling et al. 2016). We obtained MUSE observations on 2019 May 11 and October 10 as a part of the All-weather MUse Supernova Integral field Nearby Galaxies (AMUSING, Galbany et al. 2016b). The observations were taken at around 520 and $671 \mathrm{~d}$ from explosion and the SN was still detected. Fig. 1 (top panel) shows the synthetic $r$-band image of the host galaxy of SN 2017ivv at 520 d. In the bottom panel, the spectrum of the H II region near SN 2017ivv position is shown. The spectrum reveals emission lines of $\mathrm{H} \alpha, \mathrm{H} \beta$, [O III] $\lambda 4959$, [O III] $\lambda 5007$, [N II] $\lambda 6548$, [N II] $\lambda 6583$, [S II] $\lambda 6717$, and $[\mathrm{S}$ II] $\lambda 6731$ at a redshift of 0.0056 . Due to the lack of independent measurements of distance, we assume that the recession velocity is all due to cosmological expansion. However, to estimate the uncertainty in the measurements, we use a peculiar velocity of $\pm 200 \mathrm{~km} \mathrm{~s}^{-1}$ (Tully et al. 2013). With these values, we compute a distance of $d=24.09 \pm 2.90 \mathrm{Mpc}$, which corresponds to a distance modulus of $\mu=31.91_{-0.28}^{+0.25}$ mag.

The Galactic reddening in the direction of SN 2017ivv is $E(B-$ $V)=0.05 \mathrm{mag}$ (Schlafly \& Finkbeiner 2011). Due to the absence of narrow interstellar Na ID lines $(\lambda \lambda 5889,5895)$ in the SN spectra (see Section 5) and the low luminosity of the host, we assume negligible host reddening. Given the synthetic $r$-band magnitude measured from the MUSE spectrum $(r=21.74 \pm 0.19 \mathrm{mag})$, the Galactic reddening and the distance modulus, we obtain a host galaxy absolute $r$-band magnitude of $-10.3 \mathrm{mag}\left(M_{\mathrm{g}}=-10.8 \mathrm{mag}\right.$, using the Pan-STARRS

\footnotetext{
${ }^{2}$ http://wiserep.weizmann.ac.il/home
} 

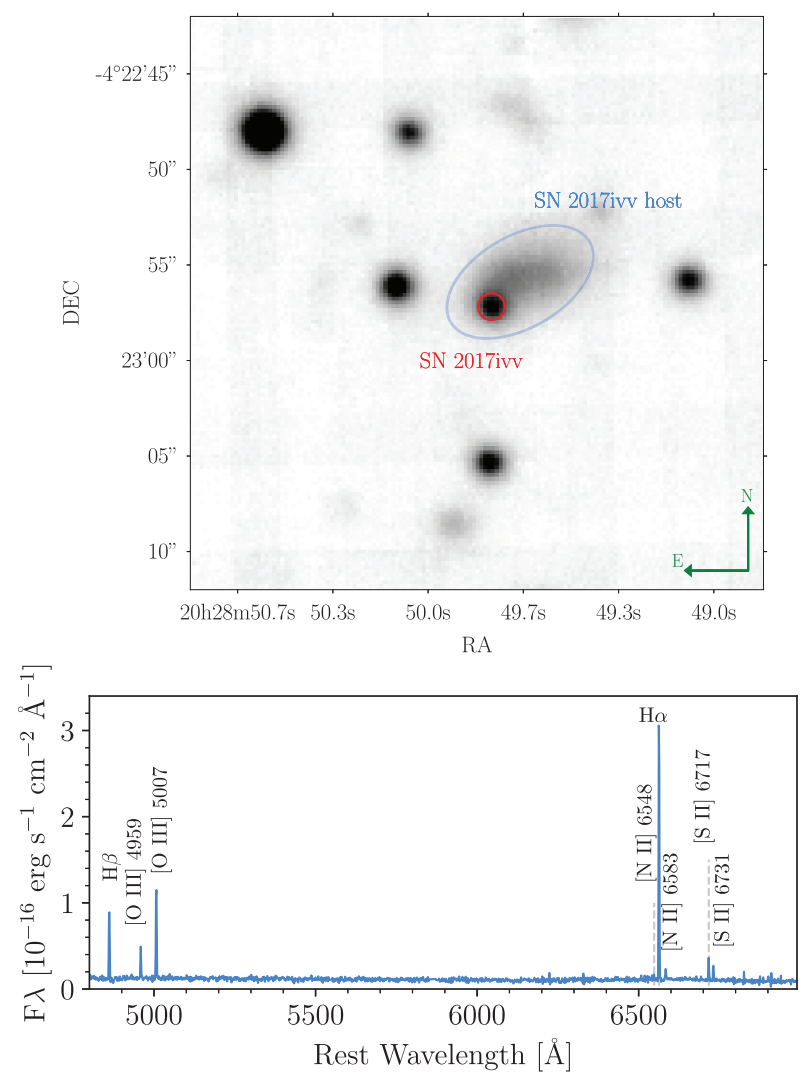

Figure 1. Top panel: MUSE image of SN 2017ivv and its host galaxy. This synthetic image is created by collapsing the data cube across the full wavelength range of the observation. Orientation is North-up, East-left. The red circle marks the $\mathrm{SN}$, while the sky-blue ellipse marks its host galaxy. Bottom panel: MUSE optical spectrum for the HII region of SN 2017ivv host. The brightest optical emission lines are labelled. Observations were obtained on 2019 May 11.

magnitude). This extremely low luminosity makes GALEXASC J202849.46-042255.5 the faintest host for a SN II to date. Using the spectrum of the H II region near SN 2017ivv, we estimate the oxygen abundance and the star formation rate (SFR). Measuring the fluxes of $\mathrm{H} \alpha, \mathrm{H} \beta$, [O III] $\lambda 5007$, and [N II] $\lambda 6583$ and applying the O3N2 and N2 diagnostic methods from Marino et al. (2013), we obtain an oxygen abundance of $12+\log (\mathrm{O} / \mathrm{H})=8.247 \pm 0.066$ dex and $12+\log (\mathrm{O} / \mathrm{H})=8.131 \pm 0.056 \mathrm{dex}$, respectively. With these values, the host galaxy of SN 2017ivv is in the lowest $\sim 3$ and $\sim 17$ per cent of the sample analysed by Anderson et al. (2016).

The SFR can be calculated using the extinction corrected luminosity of the $\mathrm{H} \alpha$ emission, $L(\mathrm{H} \alpha)$, and the normalizations from Kennicutt \& Evans (2012). For $L(\mathrm{H} \alpha)=1.089 \times 10^{38} \mathrm{erg} \mathrm{s}^{-1}$, we derive $\mathrm{SFR}=5.85 \times 10^{-4} \mathrm{M}_{\odot} \mathrm{yr}^{-1}\left[\log (\mathrm{SFR})=-3.05 \mathrm{M}_{\odot} \mathrm{yr}^{-1}\right]$, which is much smaller than the average value of the Milky Way $\left(\mathrm{SFR}=1.9 \pm 0.4 \mathrm{M}_{\odot} \mathrm{yr}^{-1}\right.$; Chomiuk \& Povich 2011).

We obtained grizy photometry of the host galaxy from the PanSTARRS1 (PS1) public science archive. ${ }^{3}$ To obtain estimates of the stellar mass $\left(M_{*}\right)$ and SFR, we fit the broadband SED from this photometry with templates from the single stellar population models of Bruzual \& Charlot (2003) via the CIGALE code (Boquien et al. 2019). We assume a Chabrier (2003) initial mass function (IMF). The

\footnotetext{
${ }^{3}$ https://catalogs.mast.stsci.edu/panstarrs/
}

best-fitting templates correspond to $M_{*}=\log \left(M / \mathrm{M}_{\odot}\right)=5.93 \pm 0.27$ and $\log (\mathrm{SFR})=-3.22 \pm 0.2 \mathrm{M}_{\odot} \mathrm{yr}^{-1}$. While these uncertainties are likely underestimated, we note that the SFR matches that measured from the $\mathrm{H} \alpha$ luminosity. This SFR corresponds to a specific SFR (sSFR) of $\log \left(\mathrm{sSFR} / \mathrm{yr}^{-1}\right)=-9.16_{0.48}^{0.46}$, which is typical of starforming galaxies and other SN II hosts (i.e. this is not a star-burst; see e.g. Taggart \& Perley 2019).

\section{PHOTOMETRIC PROPERTIES}

\subsection{Light-curve evolution}

The multiband light curves of SN 2017ivv are presented in Fig. 2. A high cadence over the first $20 \mathrm{~d}$ allows us to constrain the magnitude at maximum and the rise time. Using a polynomial fit, we find that the maximum occurs at $\sim 6$ ( $o$-band) and 8 days ( $g$ band) from the explosion at $\mathrm{M}_{o}^{\max }=-17.63 \mathrm{mag}$ and $\mathrm{M}_{g}^{\max }=-17.84 \mathrm{mag}$, respectively. This rise is consistent with the distribution of fast risetimes (7.5 \pm 0.3 days) found by González-Gaitán et al. (2015) for hydrogen-rich SNe. After the peak, a rapid decline is observed in all bands. Following the prescriptions of Anderson et al. (2014b), we find a decline rate of $7.94 \pm 0.48 \mathrm{mag}(100 \mathrm{~d})^{-1}$ in the $V$-band. Comparing this decline rate with values from the literature, we find that SN 2017ivv has the fastest initial decline measured of any SN II (Gutiérrez et al. 2017a), but it is a typical decline rate for a SN IIb [5-9 mag $(100 \mathrm{~d})^{-1}$ after peak].

Unfortunately, due to a gap in observations between 20 and $100 \mathrm{~d}$, the transition from the photospheric to the nebular phase was missed. The SN was observable again at around $100 \mathrm{~d}$ post-explosion and observed in the $B g V r i$ filters. The slope in the radioactive tail can be split into two phases, one between 100 and $350 \mathrm{~d}$, and a second one after $400 \mathrm{~d}$. For the first time period, we obtain a value of $1.36 \pm 0.02$ mag $(100 \mathrm{~d})^{-1}$ in $V$ band and $1.33 \pm 0.01 \mathrm{mag}(100 \mathrm{~d})^{-1}$ in $r$ band. For the second interval, the decay is $0.14 \pm 0.13 \mathrm{mag}(100 \mathrm{~d})^{-1}$ in the $V$ band, and $0.34 \pm 0.10 \mathrm{mag}(100 \mathrm{~d})^{-1}$ in the $r$ band. The slope between 100 and $350 \mathrm{~d}$ is larger than expected for the full-trapping of gamma-ray photons from the decay of ${ }^{56} \mathrm{Co}(0.98$ mag per 100 days in the $V$ band; Woosley, Hartmann \& Pinto 1989), suggesting that SN 2017ivv has low ejecta mass (Anderson et al. 2014b), but after $400 \mathrm{~d}$ the slope is smaller, which could be caused by some interaction between the ejecta and circumstellar material (CSM).

The high decline rate in the photospheric phase suggests that SN 2017ivv could be either a fast-declining SN II or a SNe IIb. For this reason, we will compare SN 2017ivv to both fast-declining $\mathrm{SNe}$ II and SNe IIb with well-sampled light curves and some spectra during both the photospheric and nebular phase. The fast-declining SN II group consists of ASASSN-15oz (Bostroem et al. 2019), SN 2014G (Terreran et al. 2016), SN 2013ej (Valenti et al. 2014; Huang et al. 2015; Yuan et al. 2016), and SN 2013by (Valenti et al. 2015; Black et al. 2017), while the SNe IIb sample consists of SN 2015as (Gangopadhyay et al. 2018), SN 2013df (MoralesGaroffolo et al. 2014; Szalai et al. 2016), SN 2011hs (Bufano et al. 2014), SN 2011dh (Arcavi et al. 2011; Sahu et al. 2013; Ergon et al. 2014, 2015), SN 2008ax (Pastorello et al. 2008; Taubenberger et al. 2011; Modjaz et al. 2014), and SN 1993J (Barbon et al. 1995; Matheson et al. 2000). Because of the extremely low luminosity of the host of SN 2017ivv, we also include SN 2015bs (Anderson et al. 2018), which is a slow-declining SN II that exploded in the faintest host galaxy found before SN 2017 ivv.

In Fig. 3 (top panel), we compare the $V$-band light curve of SN 2017ivv with the Type II sample. The comparison shows that SN 2017ivv has a peak luminosity similar to SN 2014G and 

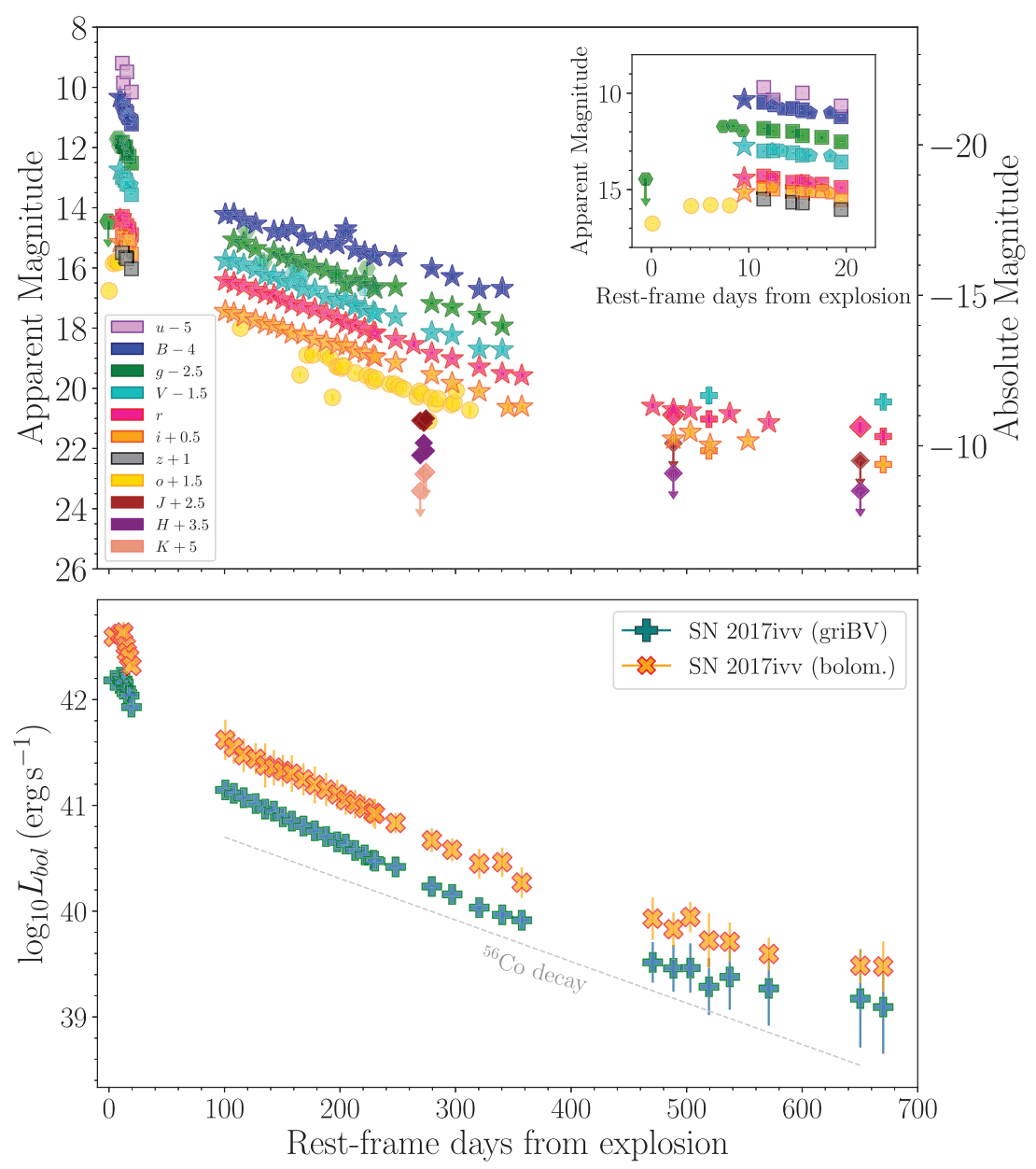

Figure 2. Upper: Optical light curves of SN 2017ivv. ATLAS photometry is presented as circles, Las Cumbres Observatory photometry as stars, Asiago photometry as squares, ASAS-SN photometry as hexagons, Post photometry as pentagons, GROND photometry as diamonds, and MUSE synthetic photometry as crosses. The photometry is corrected for MW extinction. Note that the transition between the photospheric and nebular phase happened when the supernova was behind the Sun. The inset shows the light curves around the peak. Bottom: Bolometric (orange) and pseudo-bolometric (dark cyan) light curves of SN 2017ivv.

SN 2013ej. Despite the gap during the photospheric phase, it is possible to measure the drop in luminosity from the peak to the nebular phase (100 days). For SN 2017ivv, the luminosity drops by $\lesssim 3$ mag, while SN 2014G, SN 2013ej and SN 2013by decrease by more than $4 \mathrm{mag}$. During the nebular phase, SN 2017ivv has a luminosity comparable to ASASSN-15oz. In terms of slope, it has a similar decline rate to SN 2013ej and ASASSN-15oz.

In the bottom panel of Fig. 3, the $V$-band light curve of SN 2017ivv is compared with $\mathrm{SNe} \mathrm{IIb}$. Because of the good constraint on the explosion epoch (see Fig. 2), we could confirm that the early narrow peak observed in SN 1993J and SN 2013df is not seen in SN 2017ivv (SN 2008ax does not have it either). This peak has been attributed to the cooling of the ejecta after the shock breakout (e.g. Woosley et al. 1994; Bersten et al. 2012; Nakar \& Piro 2014). SN 2017ivv displays the shortest rise-time to peak ( $\sim 6-8 \mathrm{~d})$ and is also the most luminous object $\left(\mathrm{M}_{V}=-17.67 \mathrm{mag}\right)$ among the $\mathrm{SN}$ IIb comparison sample. Its luminosity is a slightly higher than SN 2008ax and SN 1993J, which peaked at $\mathrm{M}_{V}=-17.61 \mathrm{mag}$, and $\mathrm{M}_{V}=-17.59 \mathrm{mag}$, respectively. After $150 \mathrm{~d}$, the decline rate of SN 2017ivv is slower than all $\mathrm{SNe}$ IIb in the comparison sample. This slow decline indicates that the gamma escape in SN 2017ivv is less strong, implying a higher ejecta mass.

\subsection{Bolometric luminosity}

To calculate the pseudo-bolometric luminosity, we use the extinction corrected $\mathrm{Bg}$ Vri bands converted to fluxes at the effective wavelength for each filter. We integrate a spectral energy distribution (SED) over the wavelength range of the filters, assuming zero flux at the limits. The emitted fluxes were computed at epochs when BgVri were observed simultaneously. When observations in a band were unavailable for a given epoch, the magnitudes were obtained by interpolating or extrapolating the light curves using low-order polynomials or assuming constant colours. Fluxes were converted to luminosity using the distance adopted in Section 3. As an estimate of the full bolometric light curve, we extrapolated the SED constructed from the BgVri bands using a blackbody fit to the SED. With a polynomial fit, we find a peak luminosity of $L_{\mathrm{bol}}=4.4 \times 10^{42}$ $\mathrm{erg} \mathrm{s}^{-1}$ occurring at $\sim 10.5 \pm 2 \mathrm{~d}$ after explosion $(\mathrm{MJD}=58102.18$ ). This maximum is about $\sim 2.5-4.5 \mathrm{~d}$ later than the peaks in the $o$ and $g$ bands. After peak, the light curve declines at a rate of 7.97 mag per $100 \mathrm{~d}$, quite similar to the $V$ band. The slope in the tail is $1.31 \mathrm{mag}(100 \mathrm{~d})^{-1}$, a slightly smaller than the $V$-band slope of [1.36 mag $\left.(100 \mathrm{~d})^{-1}\right]$. These rapid declines suggest that the gamma-ray trapping is incomplete. 


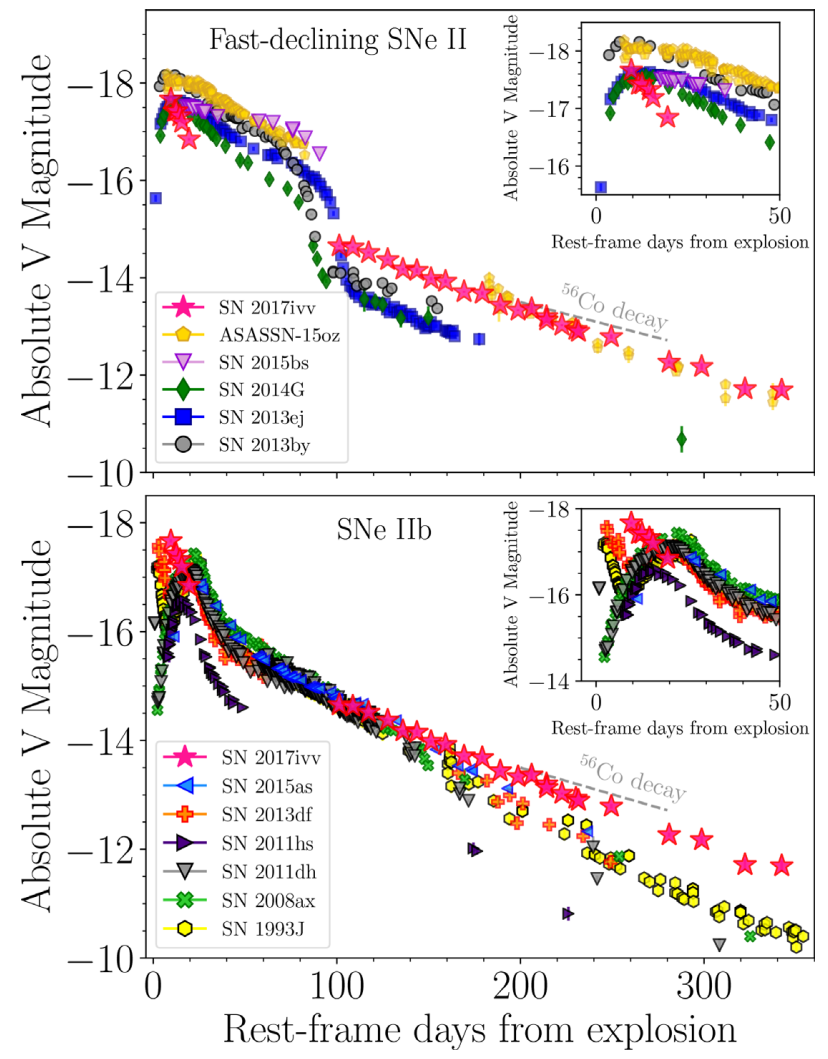

Figure 3. Photometric comparison of SN 2017ivv with the fast-declining SN II (top) and SN IIb (bottom) samples. The extinction and distance assumptions to calculate the absolute magnitudes were adopted from the references in Section 4.1.

The ${ }^{56} \mathrm{Ni}$ mass can estimated by comparing the bolometric luminosity of SN 2017ivv with the theoretical values of full ${ }^{56} \mathrm{Co}$ deposition, as reported by Jerkstrand et al. (2012):

$L_{0}(t)=9.92 \times 10^{41} \frac{M\left({ }^{56} \mathrm{Ni}\right)}{0.07 \mathrm{M}_{\odot}}\left(\mathrm{e}^{-t / 111.4}-\mathrm{e}^{-t / 8.8}\right) \mathrm{erg} \mathrm{s}^{-1}$,

where $M\left({ }^{56} \mathrm{Ni}\right)$ is the mass of ${ }^{56} \mathrm{Ni}$ synthesized during the explosion. As the light curve tail does not follow the ${ }^{56} \mathrm{Co}$ decay, the gamma escapes needs to be considered (Clocchiatti \& Wheeler 1997; Jerkstrand 2017) as follows:

$L(t)=L_{0}(t) \times\left(1-\mathrm{e}^{-\left(\tau_{t r} / t\right)^{2}}\right) \operatorname{erg~\mathrm {s}^{-1}}$,

where $L_{0}$ comes from equation (1) and $\tau_{\text {tr }}$ is the full-trapping characteristic time-scale. We note that equation (2) is appropriate as long as positron contributions are minor (likely before $350 \mathrm{~d}$ ). Using these equations to fit the bolometric light curve of SN 2017ivv between 120 and $350 \mathrm{~d}$, we find a nickel mass of $\mathrm{M}\left({ }^{56} \mathrm{Ni}\right)=$ $0.059 \pm 0.003 \mathrm{M}_{\odot}$ and $\tau_{\text {tr }}=298 \pm 8 \mathrm{~d}$.

\subsection{Evolution of the colour curves}

Fig. 4 compares the $(B-V)$ and $(V-i)$ colours of SN 2017ivv with the fast-declining sample and SN 2015bs (left-hand panel) and with $\mathrm{SNe}$ IIb (right-hand panel). In the former, the behaviour of all $\mathrm{SNe}$ at early phases is quite similar. At late phases, the colour evolution of SN 2017ivv is comparable to ASASSN-15oz. Unfortunately, the other objects have no colour information after $100 \mathrm{~d}$. Nevertheless, the overall colour evolution of SN 2017ivv seems similar to that observed in the fast-declining objects.

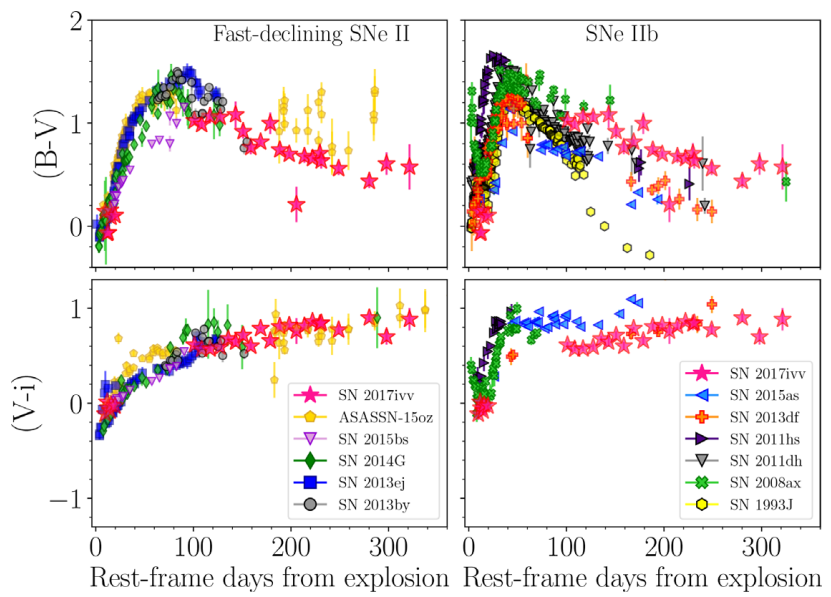

Figure 4. Colour evolution of SN 2017ivv compared to fast-declining SNe II (left-hand panels) and SNe IIb (right-hand panels). See Section 4.1 for the references.

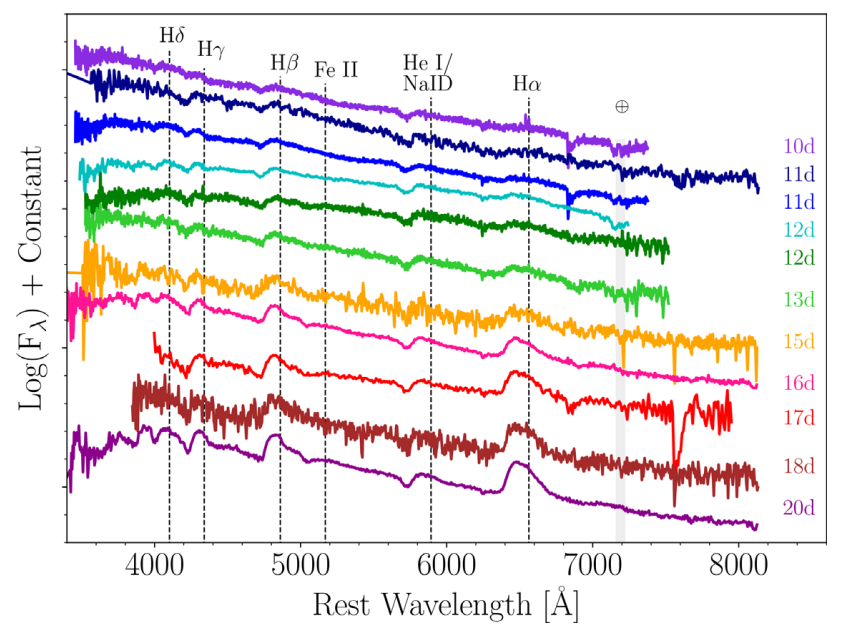

Figure 5. Optical spectra of SN 2017ivv ranging from 10 to $20 \mathrm{~d}$ from explosion. The phases are labelled on the right. Each spectrum has been corrected for Milky Way reddening and shifted in flux for comparison.

SN 2017ivv is bluer at early times than the SNe IIb sample. Then, it rapidly becomes redder. Between 100 and $150 \mathrm{~d}$, the $(B-V)$ evolution is nearly similar to that of SN 2008ax, although it is redder than most of the comparison sample. After $150 \mathrm{~d}$, it evolves back to bluer colours. At epochs later than $100 \mathrm{~d}$, the $(V-i)$ colours of SN 2017ivv are similar to those of SN 2013df.

\section{SPECTRAL PROPERTIES}

\subsection{Early phases}

Fig. 5 shows the optical spectra of SN 2017ivv from 10 to $20 \mathrm{~d}$. The early spectra display a blue continuum, with low-contrast hydrogen and $\mathrm{He}$ I $\lambda 5876$ lines that are typical of young SNe II. At $16 \mathrm{~d}$, these lines become stronger, and broad P-Cygni profiles are visible. In particular, $\mathrm{H} \alpha$ exhibits a broad and asymmetric emission component with an extremely weak absorption feature. Weak absorption profiles have been seen in fast-declining SNe II and interpreted as evidence for a low amount of absorbing material along the line of sight due to either a low-mass envelope or a very steep density gradient (e.g. Schlegel 1996; Gutiérrez et al. 2014). Asymmetric emission 

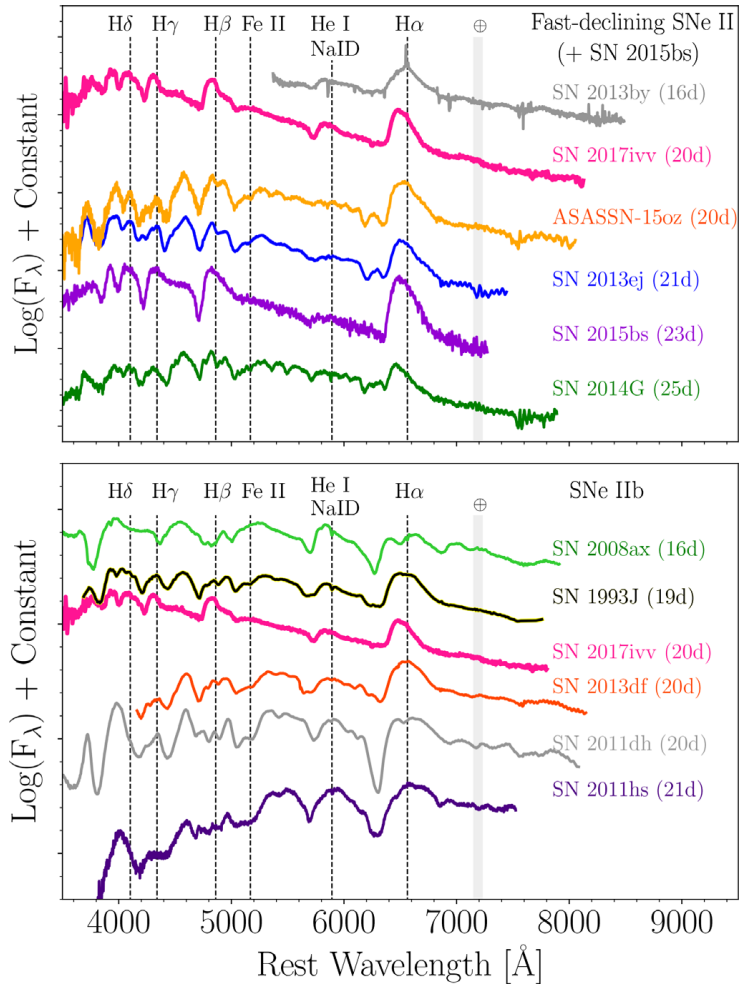

Figure 6. Spectral comparison at $\sim 20 \mathrm{~d}$ of $\mathrm{SN} 2017 \mathrm{ivv}$ with the fastdeclining SNe II (top panel) and SNe IIb (bottom panel). The spectra have been corrected by Milky Way reddening and redshift.

profiles are also detected in $\mathrm{H} \beta$. Between 16 and $20 \mathrm{~d}$, the emission component of $\mathrm{H} \alpha$ shows a flat-topped profile indicating either a weak interaction with a low-density CSM (e.g. Inserra et al. 2011) or the presence of an expanding, geometrically thin $\mathrm{H}$ shell (Barbon et al. 1995). Similar profiles have been seen before in well-studied SNe II at various phases, e.g. SN 2007od (Inserra et al. 2011; Gutiérrez et al. 2017a); SN 2007X (Gutiérrez et al. 2017a); SN 2016esw (de Jaeger et al. 2018); and SNe IIb, e.g. SN 1993J (Barbon et al. 1995; Matheson et al. 2000); SN 2011hs (Bufano et al. 2014). After 20 d, Fe II $\lambda 5169$ starts to be visible. No narrow Na ID $(\lambda \lambda 5889,5895)$ absorption features from either the host galaxy or the Milky Way are detected, suggesting little reddening in the direction of the SN.

Overall, the line profiles of SN 2017ivv display blue-shifted emission-line peaks. In the case of $\mathrm{H} \alpha$, the velocity offset of the emission component is $\sim 3300 \mathrm{~km} \mathrm{~s}^{-1}$. For $\mathrm{H} \beta$, the velocity offset is $\sim 2000 \mathrm{~km} \mathrm{~s}^{-1}$, while for He I/Na ID, it is between 2200 and $2700 \mathrm{~km} \mathrm{~s}^{-1}$. According to Anderson et al. (2014a), such blueshifted velocity offsets are common properties of SN II spectra and are caused by the density distribution of the ejecta.

In Fig. 6, the spectrum of SN 2017ivv at $20 \mathrm{~d}$ from explosion is compared with the fast-declining SNe II (top panel) and SNe IIb (bottom panel) samples at a similar epoch. SN 2017ivv has a peculiar $\mathrm{H} \alpha$ P-Cygni profile compared with the fast-declining objects. While ASASSN-15oz, SN 2013ej and SN 2014G show a small absorption component together with the Cachito line (Gutiérrez et al. 2017a), SN 2017ivv and SN 2013by display a flat absorption component. Although they have similar absorption profiles, the emission components are different. For SN 2013by the emission is broader and symmetric compared to SN 2017ivv. The slowdeclining SN II 2015bs, also included in this comparison, shows many similarities with SN 2017ivv: they only display hydrogen lines and $\mathrm{He} \mathrm{I} / \mathrm{NaI} \mathrm{D}$, the $\mathrm{H} \alpha$ profile shows an asymmetric emission and flat absorption, while Fe II $\lambda 5169$ starts to be visible. From the comparison with the $\mathrm{SNe} \mathrm{IIb}$, the boxy $\mathrm{H} \alpha$ profile observed in SN 2017ivv is similar to that of SN 1993J. This profile shape has been attributed to ejecta asymmetry, two line-forming regions for hydrogen, or CSM interaction (e.g. Baron et al. 1995; Hachinger et al. 2012). The other objects have a $\mathrm{H} \alpha$ emission, with some signs of helium. In general, the bluer part of the spectrum of SN 2017ivv (below $5000 \AA$ ) shows considerably fewer lines than fast-declining SNe II and SNe IIb. This suggests that SN 2017ivv has either a higher temperature than the other $\mathrm{SNe}$ or the appearance of the lines is a metallicity effect (Dessart et al. 2014; Anderson et al. 2016; Gutiérrez et al. 2018). We note that the latter alternative is consistent to the low-metallicity of the host (see Section 3).

\subsection{Late phases}

In the nebular phase, we obtained 14 spectra of SN 2017ivv between 110 and $671 \mathrm{~d}$ (Fig. 7). During this phase, the spectra are mainly dominated by forbidden emission lines formed in low density regions. At $110 \mathrm{~d}, \mathrm{H} \alpha$ and the Ca II NIR triplet are the strongest features in the spectrum. The forbidden lines of [O I] $\lambda 5577,[\mathrm{OI}]$ $\lambda \lambda 6300,6364$ and [CaII] $\lambda \lambda 7291,7324$ are also detected, but a slightly weaker. At bluer wavelengths, the iron absorption lines are still dominating the spectrum. The Na ID doublet displays a clear residual absorption component. This surviving P-Cygni profile is visible until $\sim 250 \mathrm{~d}$. Between 110 and $158 \mathrm{~d}$, the spectra show only minor evolution, with the emission lines increasing gradually in strength relative to the continuum.

From 158 to $242 \mathrm{~d}$, the strength of the emission lines completely changes. At $158 \mathrm{~d}, \mathrm{H} \alpha$ is the strongest line in the spectrum, followed by the Ca II NIR triplet and [Ca II] $\lambda \lambda 7291,7324$. However, after $242 \mathrm{~d}$, [Ca II] becomes the strongest feature. The intensity of $\mathrm{H} \alpha$ decreases while [OI] $\lambda \lambda 6300,6364$ grows. At $242 d$, [O I] $\lambda 5577$ emission is barely detected, $\mathrm{Mg} \mathrm{I}]$ and [Fe II] $\lambda \lambda 7155,7172$ start to be detectable and the Ca II NIR triplet diminishes. We also notice that $\mathrm{H} \alpha$ develops a 'flat-topped' emission profile (the right-hand panel of Fig. 7). $\mathrm{H} \alpha$ starts to have a 'bridge' with [OI] $\lambda \lambda 6300,6364$. This 'bridge' has been seen before in both SNe IIL (e.g. 2013by; Black et al. 2017 and 2014G; Terreran et al. 2016) and SNe IIb (e.g. SN 1993J; Matheson et al. 2000). From 337 to 671 d, the strength of [Ca II] and [O I] lines decrease dramatically, while the $\mathrm{H} \alpha$ emission line becomes much broader; $\mathrm{H} \alpha$ is the dominant feature and the 'bridge' with [O I] disappears. More details about the evolution of the nebular lines in SN 2017ivv and their connection with the SN IIb class can be found in Section 6.2.

A comparison of the nebular spectrum of SN 2017ivv at 158 and $337 \mathrm{~d}$ with spectra for the fast-declining sample and SN 2015bs at similar epochs is presented in Fig. 8 (left-hand panels). At around $158 \mathrm{~d}$ (top panel), all $\mathrm{SNe}$ show similar spectra, with $\mathrm{H} \alpha$ being the dominant feature, followed by the Ca II NIR triplet. One obvious feature observed in SN 2017ivv and missing in the other SNe is the blue-shifted of the emission-line peaks. Blue-shifted lines can indicate asymmetries in the ejecta or radiative transfer effects (see more details in Section 6.2). At around $338 \mathrm{~d}$ (bottom panel), SN 2017ivv has unusual strengths in its emission lines. While $\mathrm{H} \alpha$ is the dominant feature in the fast-declining objects, in SN 2017ivv $\mathrm{H} \alpha$ is weak and [Ca II] is the strongest line. For the slow-declining SN II 2015bs, the strength of $\mathrm{H} \alpha$, [Ca II], and [O I] seem to be similar (see Section 6.1). Another peculiar characteristic of SN 2017ivv is the strength of the [O I] $\lambda \lambda 6300,6364$ doublet. According to Jerkstrand et al. (2014), [O I] $\lambda 6364$ is always equivalent (thick limit) or weaker 


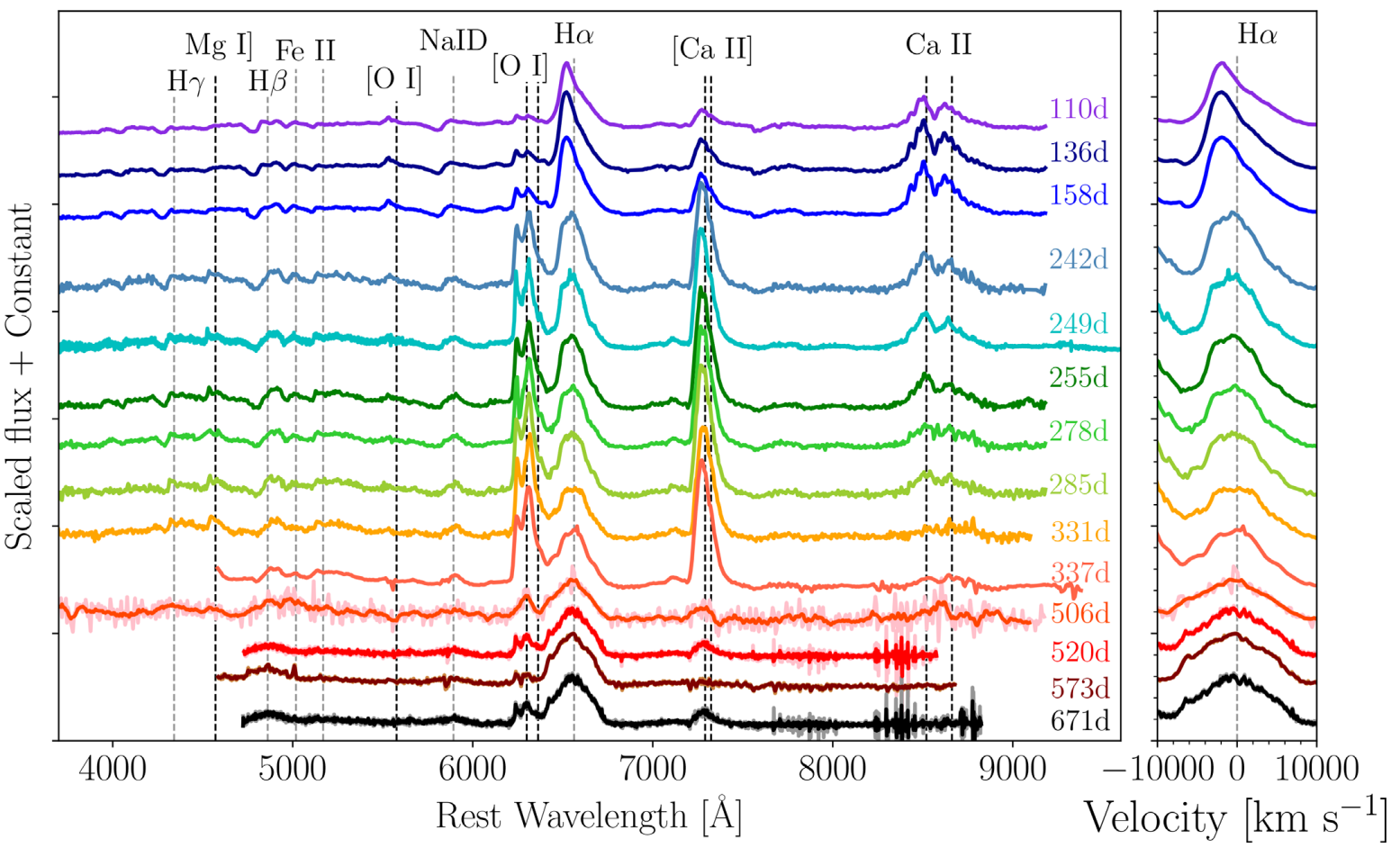

Figure 7. Left: Optical spectra of SN 2017ivv ranging from 110 to $671 \mathrm{~d}$ from explosion. The phases are labeled on the right. Each spectrum has been corrected for Milky Way reddening and shifted by an arbitrary amount for presentation. Right: Zoom around the H $\alpha$ P-Cygni profile in velocity space.
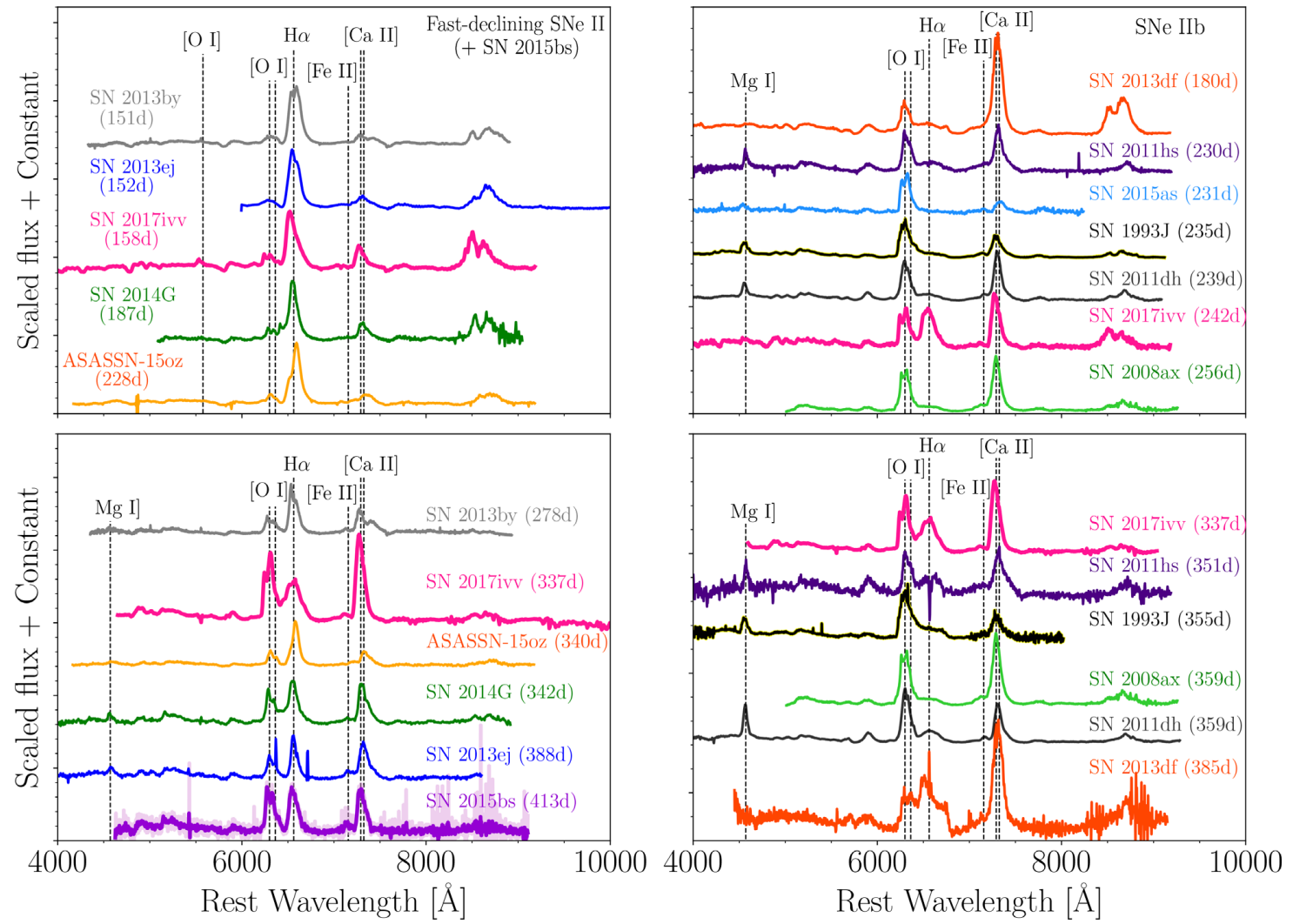

Figure 8. Spectral comparison of SN 2017ivv in nebular phases. Left-hand panels: Comparisons at $\sim 158$ (top panel) and $\sim 337 \mathrm{~d}$ (bottom panel) with the fast-declining SN II sample and SN 2015bs at similar epochs. Spectra are scaled to match H $\alpha$ and shifted for presentation. Right-hand panels: Comparisons at $\sim 242$ (top panel) and $\sim 337 \mathrm{~d}$ (bottom panel) with the SN IIb sample at similar epochs. Spectra are scaled to match [O I] and shifted for presentation. All spectra have been corrected by Milky Way reddening and redshift. 
(thin limit) than [O I] $\lambda 6300$. In SN 2017ivv, however, the relative strengths of the [OI] doublet lines appear inverted. More details about this line is presented in Section 6.2.2.

In Fig. 8 (right-hand panels), SN 2017ivv is compared with the SN IIb sample at around 242 and $337 \mathrm{~d}$. At $~ 242 \mathrm{~d}$ (top panel), all SNe show the [O I] doublet feature with a blue-shifted emission peak. This blue-shift disappears in SN 2011hs and SN 2013df $100 \mathrm{~d}$ later (bottom panel). [Ca II] is blue-shifted in SN 1993J, SN 2008ax and SN 2017ivv, red-shifted in SN 2015as, and at the rest in SN 2011hs and SN 2011dh. At $337 \mathrm{~d}$, the only object with [Ca II] still blue-shifted is SN 2017ivv. Overall, two evident differences between SN 2017ivv and the sample of SN IIb are the strength of Mg I], which is weaker in the spectra of SN 2017ivv, and the strong and broad $\mathrm{H} \alpha$ emission profile in SN 2017ivv.

A broad $\mathrm{H} \alpha$ emission profile has been identified in the latetime spectra of SNe IIb (e.g. SN 1993J, SN 2008ax, SN 2011dh, SN 2011hs, SN 2013dh; see bottom panel) and has been linked to CSM interaction (e.g. Patat, Chugai \& Mazzali 1995; Matheson et al. 2000; Taubenberger et al. 2011; Maeda et al. 2015). Alternatively, Maurer et al. (2010) explain the presence of $\mathrm{H} \alpha$ as the result of mixed and strongly clumped $\mathrm{H}$ and $\mathrm{He}$, while $\mathrm{J} 15 \mathrm{~b}$ show that in radioactivity-powered models emission here is completely dominated by $[\mathrm{N} \mathrm{II}] \lambda \lambda 6548,6583$, with only a minor $\mathrm{H} \alpha$ contribution (see also Fang \& Maeda 2018). One should in general differentiate the early and later nebular evolution; several IIb $\mathrm{SNe}$ show consistency with [N II] in an early radioactivity-powered phase, transitioning to interaction and an $\mathrm{H} \alpha$-dominated spectrum at $\gtrsim 500-700 \mathrm{~d}$. Although $\mathrm{H} \alpha$ has been identified previously in many $\mathrm{SNe} \mathrm{IIb}$, the strength and evolution observed in SN 2017ivv are somewhat unprecedented.

\subsection{Expansion velocities}

The broad lines in the early spectra of SN 2017ivv indicate large expansion velocities. In Fig. 9 (top panel), we present the velocity evolution of $\mathrm{H} \alpha, \mathrm{H} \beta$, and $\mathrm{He}$ I $\lambda 5876$ during the first $20 \mathrm{~d}$. These velocities were mostly obtained from the minimum flux of the absorption. In the case of $\mathrm{H} \alpha$, it was derived from the full width at half-maximum (FWHM) of the emission component (see Gutiérrez et al. 2017a). All lines have nearly constant velocities, although the $\mathrm{H} \beta$ velocity seems to increase by around $1000 \mathrm{~km} \mathrm{~s}^{-1}$ from 10 to $\sim 15$ d. Folatelli et al. (2014) found similar velocity evolution for SN 2010as and some transitional Type Ib/c SNe, the so-called flatvelocity Type IIb Supernovae. The behaviour was most notable for the helium lines, but in some objects it was a general feature of the photosphere, and could be evidence of the presence of a dense shell inside the $\mathrm{SN}$ ejecta.

The time evolution of the expansion velocity of $\mathrm{H} \beta$ in SN 2017ivv is compared with the fast-declining and SN IIb samples in the middle and bottom panels of Fig. 9, respectively. The velocity evolution of SN 2017ivv during the first $16 \mathrm{~d}$ is different from all other SNe because they all have rapidly evolving velocities. After $16 \mathrm{~d}$, the fast-declining, SN 2014G, and the SNe IIb, 1993J and 2013df have similar velocities than SN 2017ivv.

During the nebular phase, the velocity of the emission lines is measured using the line FWHM. The FWHM velocities of $\mathrm{H} \alpha$; [O I] $\lambda \lambda 6300,6364$; [Ca II] $\lambda \lambda 7291,7324$; and [Fe II] $\lambda \lambda 7155,7172$ are measured by fitting Gaussian profiles to each line. When a line is affected by another one, we first remove the potential contribution of the latter and then we fit the line. Examples of Gaussian fits at four different epochs are presented in Fig. B1 (online supplementary material). Finally, all FWHMs were corrected for the resolution of the instrument following the prescriptions of Maguire et al. (2012). The
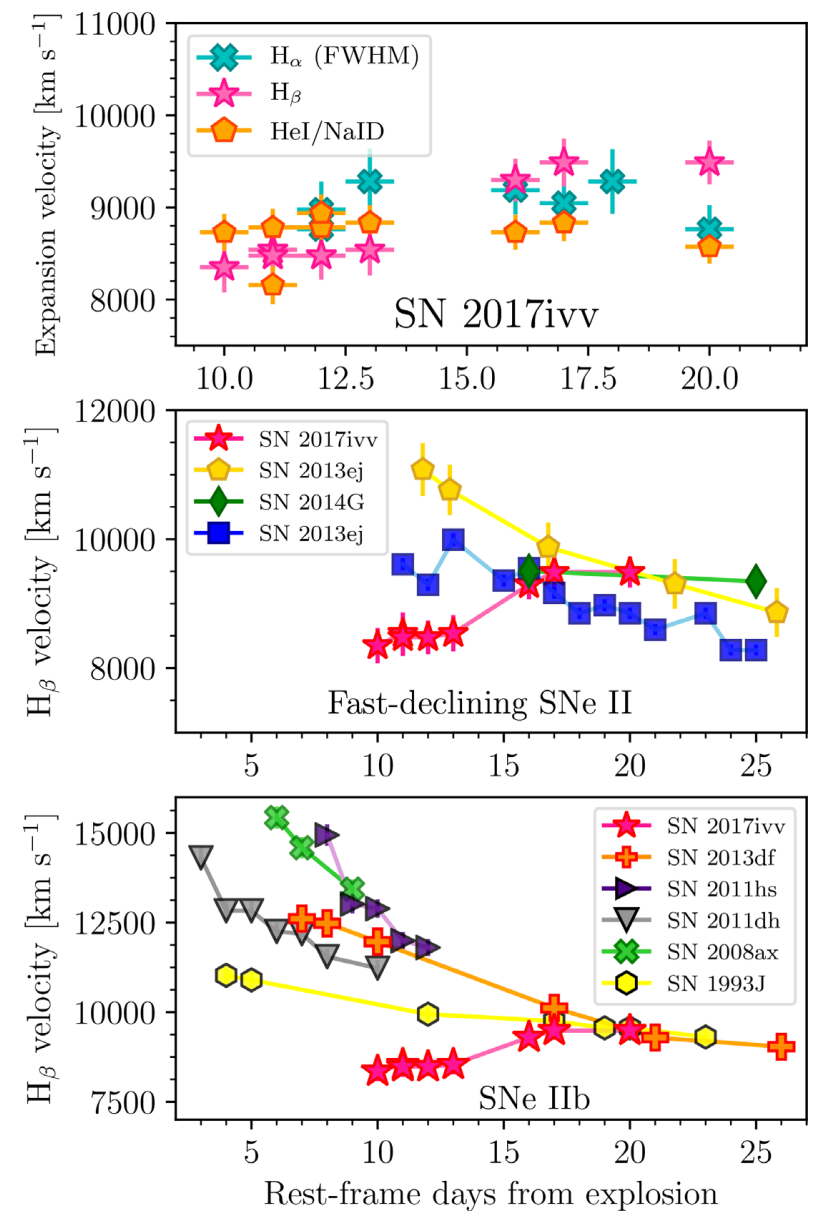

Figure 9. Top panel: Expansion velocity evolution of SN 2017ivv over the first $20 \mathrm{~d}$. The $\mathrm{H} \alpha$ velocity was obtained from the FWHM of the emission line, while $\mathrm{H} \beta$ and $\mathrm{He}$ I were derived from the minimum of the absorption line. Middle panel: Comparison of the $\mathrm{H} \beta$ velocity of SN 2017ivv with the fast-declining sample. Bottom panel: Comparison of the $\mathrm{H} \beta$ velocity of SN 2017ivv with SN IIb sample.

FWHM velocities are reported in Table A9 (online supplementary material) and shown in the top panel of Fig. 10. Here, we see that [O I], [Ca II], and [Fe II] show little velocity evolution. After $337 \mathrm{~d}$, [Fe II] is barely detected, so no estimation of its velocity is presented. Flat FWHM evolution in SNe II has been reported before by Maguire et al. (2012), Silverman et al. (2017) although only after $~ 300 \mathrm{~d}$. Before $300 \mathrm{~d}$, decreasing velocities were measured.

SN 2017ivv has an $\mathrm{H} \alpha$ FWHM velocity increasing with time from $\mathrm{FWHM}=5970 \mathrm{~km} \mathrm{~s}^{-1}$ at $158 \mathrm{~d}$ to FWHM $=10510 \mathrm{~km} \mathrm{~s}^{-1}$ at $671 \mathrm{~d}$. The increase in the FWHM of $\mathrm{H} \alpha$ is large (more than $4000 \mathrm{~km} \mathrm{~s}^{-1}$ in $\sim 510$ d). For $[\mathrm{O}$ I] $\lambda \lambda 6300,6364$, the FWHM velocity remains at $\sim 5000 \mathrm{~km} \mathrm{~s}^{-1}$. This FWHM value is similar to that found by Taubenberger et al. (2009) for stripped-envelope SNe, and larger than the average value for SNe II $\left(3000 \mathrm{~km} \mathrm{~s}^{-1}\right.$; Maguire et al. 2012).

On the bottom panel of Fig. 10, we present the velocity offset of the emission lines. All of the lines are blue-shifted with velocity offsets between -2000 and $-400 \mathrm{~km} \mathrm{~s}^{-1}$. The offset slowly decreases with time, but without reaching zero velocity. Generally, for opacity caused by atoms (in contrast to dust) the blue-shifts of emission peaks gradually disappear as the optical depths decrease with time (e.g. J15b). There is a weak such trend up to $300 \mathrm{~d}$ for $\mathrm{H} \alpha$, [Ca II], 

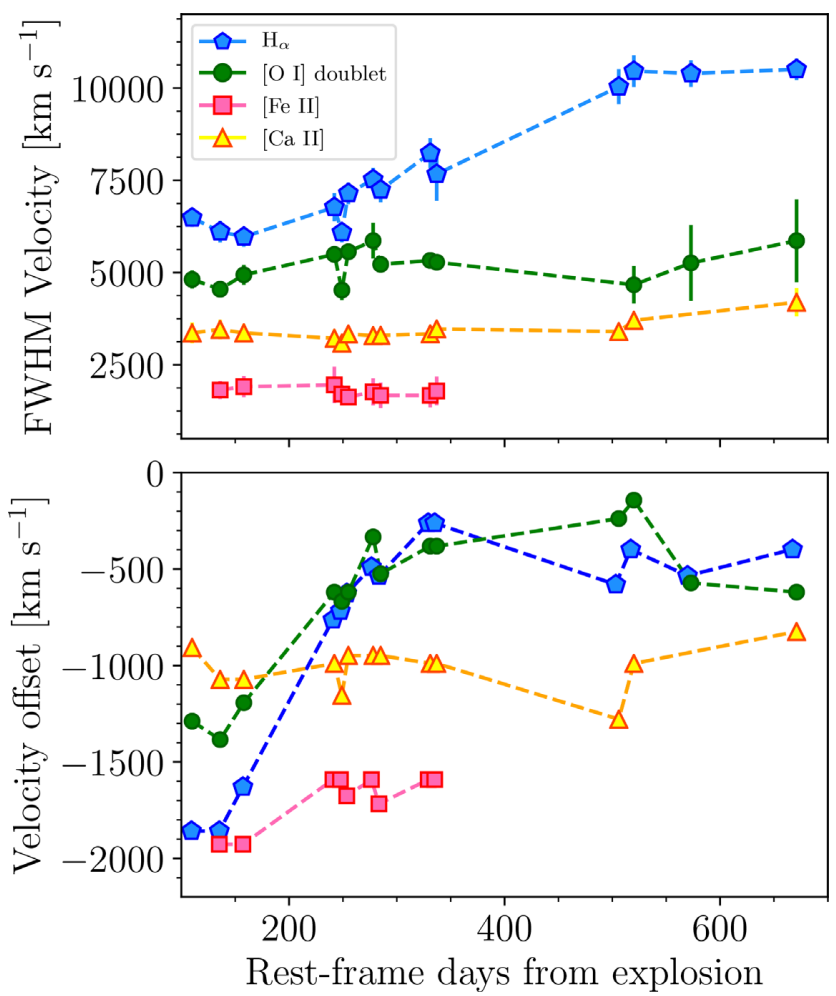

Figure 10. Top panel: FWHM velocity of $\mathrm{H} \alpha$ (orange), [O I] (blue), [Ca II] (magenta) and [Fe II] (green) emission lines of SN 2017ivv. Bottom panel: Velocity offset of emission lines in SN 2017ivv. Same colours and markers as in the top panel.

[Fe II], and the [O I] doublet taken as a whole. However, after that time the blue-shifts are retained at roughly constant values. Such levels of blue-shifts observed in SN 2017ivv have been seen before in SN 1993J and were explained by an asymmetric mass distribution for the emitting material in the envelope (Spyromilio 1994). More details can be found in Section 6.2.

\section{ANALYSIS AND DISCUSSION}

\subsection{A low-metallicity host and its effects on the SN spectra}

The host galaxy of SN 2017ivv has the lowest luminosity $\left(M_{r} \sim\right.$ $-10.3 \mathrm{mag}$, see Section 3) of any SN II host found to date. The previous record, the host of SN 2015bs was about two magnitudes more luminous (Anderson et al. 2018).

Dessart et al. $(2013,2014)$ presented SN II spectral models produced from progenitors with metallicities of $0.1,0.4,1$, and 2 times solar metallicity $\left(Z_{\odot}\right)$. Dessart et al. (2014) predicted a dependence of the strength of the metal lines observed during the recombination phase with the progenitor metallicity. Anderson et al. (2016) tested this prediction using more than 100 SNe II, and found a trend between the strength of Fe II $\lambda 5018 \AA$ and the local metallicity (oxygen abundances). Similar results were found by Taddia et al. (2016) and Gutiérrez et al. (2018).

Anderson et al. (2018) found a remarkable similarity between $\mathrm{SN} 2015 \mathrm{bs}$ at $57 \mathrm{~d}$ and the $0.1 \mathrm{Z}_{\odot}$ model at 50 days. Unfortunately, for SN 2017ivv this analysis is impossible to make due to the lack of observations between 20 and $100 \mathrm{~d}$. However, the effects of metallicity could be also be explored during the nebular phase. Through comparison to the nebular-phase spectral models, Anderson
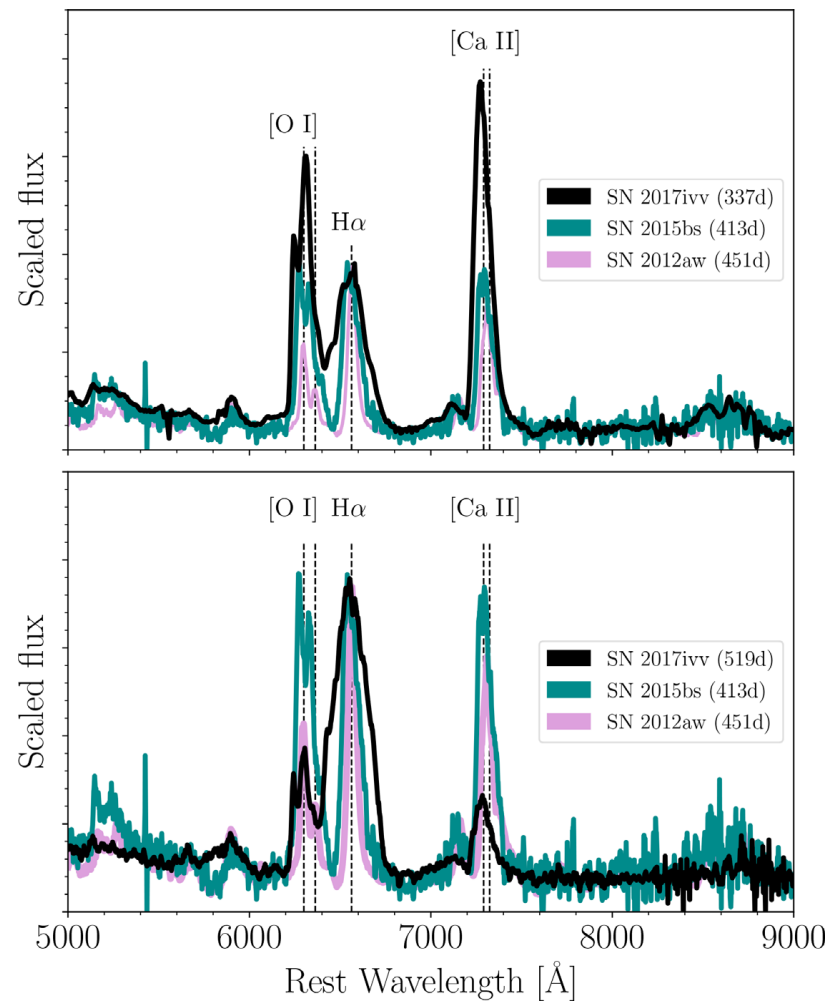

Figure 11. Spectral comparison of SN 2017ivv at 337 (top) and $519 \mathrm{~d}$ (bottom) with SN 2015 bs at 413 d. For reference, we use the spectrum of the slow-declining SN II, SN 2012aw at 451 d (Jerkstrand et al. 2014). Spectra are scaled to match the $\mathrm{H} \alpha$ line.

et al. (2018) argued that SN 2015bs has a high mass progenitor since the strength of the [OI] $\lambda \lambda 6300,6364$ was bounded by the $15 \mathrm{M}_{\odot}$ and $25 \mathrm{M}_{\odot}$ progenitor models. This result was based on the relation between the helium-core and ZAMS mass (Woosley \& Weaver 1995).

We compared the nebular spectra of SN 2017ivv and SN 2015bs in Fig. 11. Because we do not have observations at similar epochs, we use the nebular spectrum of SN 2015bs at $413 \mathrm{~d}$, and two spectra of SN 2017ivv at $337 \mathrm{~d}$ (top panel) and $519 \mathrm{~d}$ (bottom panel). In the top panel we see that the strength of the [OI] line is larger in SN $2017 \mathrm{ivv}$, with the redder peak stronger than the bluer (more details can be found in Section 6.2.2). In SN 2015bs, $\mathrm{H} \alpha$ has a narrower profile, while $\mathrm{Ca}$ II is weaker. As the [O I] doublet is a good tracer of the ZAMS progenitor mass, this comparison could imply a more massive progenitor for SN 2017ivv. However, a higher helium core mass (strong [O I]) can be also related to metallicity. Woosley et al. (2002) predict that for massive stars the helium-core mass increases as metallicity decreases. As SN 2017ivv exploded in a metal-poor environment, this possibility cannot be ruled out.

When SN 2017ivv is compared at 519 d with SN 2015bs (bottom panel), the similarities disappear. In SN 2017ivv all emission lines are less prominent than those of SN 2015bs. The changes in the emission lines and the broad profile of $\mathrm{H} \alpha$ could indicate an interaction between the ejecta and the CSM (see Section 6.2). This hypothesis is also supported by the shallow slope of the light curves at late times.

\subsection{Nebular line evolution}

One of the most interesting characteristics of SN 2017ivv is the unusual late-time spectral evolution. Fig. 12 shows the profiles of the 


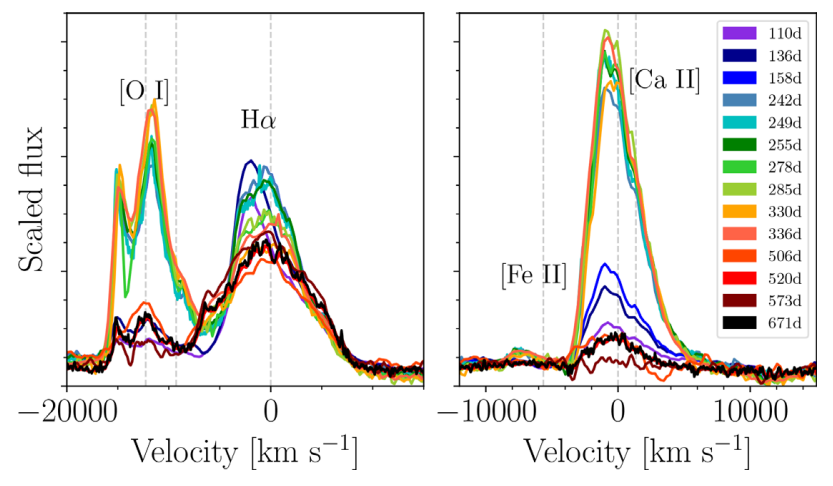

Figure 12. Observed profiles of SN 2017ivv in the nebular phase. Lefthand panel: [O I] $\lambda \lambda 6300,6364$ and $\mathrm{H} \alpha$ emission lines. Right-hand panel: [Fe II] $\lambda \lambda 7155,7172$ and [Ca II] $\lambda \lambda 7291,7324$ emission lines. The phases are labelled on the right.

$\mathrm{H} \alpha$, [O I], [Fe II] and [Ca II] lines between 110 and $671 \mathrm{~d}$. Details of the evolution of each line are presented below.

\subsubsection{Ho emission-line profile}

The evolution of the $\mathrm{H} \alpha$ emission line is shown in the left panel of Fig. 12. Overall, $\mathrm{H} \alpha$ is strongly asymmetric with a slowly dropping blue-shift in the emission peak. The velocity offset (shown in the bottom panel of Fig. 10) declines from $\sim-1860 \mathrm{~km} \mathrm{~s}^{-1}$ at $110 \mathrm{~d}$ to $\sim-400 \mathrm{~km} \mathrm{~s}^{-1}$ at $671 \mathrm{~d}$. Blue-shifted lines during the nebular phase have been related to asymmetries in the ejecta (e.g. Utrobin \& Chugai 2009) or radiative transfer effects due to dust (e.g. Sahu et al. 2006; Maguire et al. 2010, 2012) or line opacities (J15b). There are negligible changes in the intensity and width of the $\mathrm{H} \alpha$ line between 110 to 158 days. After 158 days, the $\mathrm{H} \alpha$ flux drops significantly (see Fig. 15), but the line is getting broader. Specifically, from 158 to $242 \mathrm{~d}$, the $\mathrm{H} \alpha$ flux declines around 20 per cent. Although the $\mathrm{H} \alpha$ flux is predicted to decline at late-times (e.g. Chevalier \& Fransson 1994), observations of SNe II with well-sampled nebular coverage (e.g SN 2004et, Sahu et al. 2006) have shown that this drop usually happens gradually after $\sim 300-400 \mathrm{~d}$. Similar decreases in the $\mathrm{H} \alpha$ fluxes have been seen for a few SNe II (e.g. SN 1980K; Fesen et al. 1999), but only after a few years.

After $242 \mathrm{~d}, \mathrm{H} \alpha$ develops a 'flat-topped' emission profile, has a weaker strength compared with [OI] and [CaII], and displays a different profile from that observed in $\mathrm{SNe}$ II at similar phases (Fig. 8). A and flat-topped $\mathrm{H} \alpha$ emission can be associated with CSM interaction in a roughly spherical shell (Chevalier \& Fransson 1985; Jeffery \& Branch 1990; Chevalier \& Fransson 1994), however, for SN 2017ivv, the fade and shape of $\mathrm{H} \alpha$ seem to be related to the end of the hydrogen recombination. At $242 \mathrm{~d}$, the broad $\mathrm{H} \alpha$ emission is becoming blended with [O I] $\lambda \lambda 6300,6364$. The 'bridge' between these two lines grows for more than $200 \mathrm{~d}$. In the spectrum at $506 \mathrm{~d}$, the 'bridge' begins to recede, while the $\mathrm{H} \alpha$ emission develops a blue shoulder, that makes it even broader.

Terreran et al. (2016) showed that the 'bridge' between $\mathrm{H} \alpha$ and [O I] observed in the SN 2014G can be explained in terms of boxy line profiles due to CSM interaction. Similar line structures are seen in SNe IIb such as SN 1993J, SN 2008ax, SN 2011fu, SN 2011hs, and SN 2013df. For SN 2017ivv, we reproduce the emission profiles in the 6200-6800 A region and the best fit is presented in Fig. 13. To fit this region simultaneously, we considered a two-component model for the [O I] doublet and a single Gaussian for $\mathrm{H} \alpha$. Therefore, the profile fit consists of six parts: a linear continuum; two narrow Gaussians

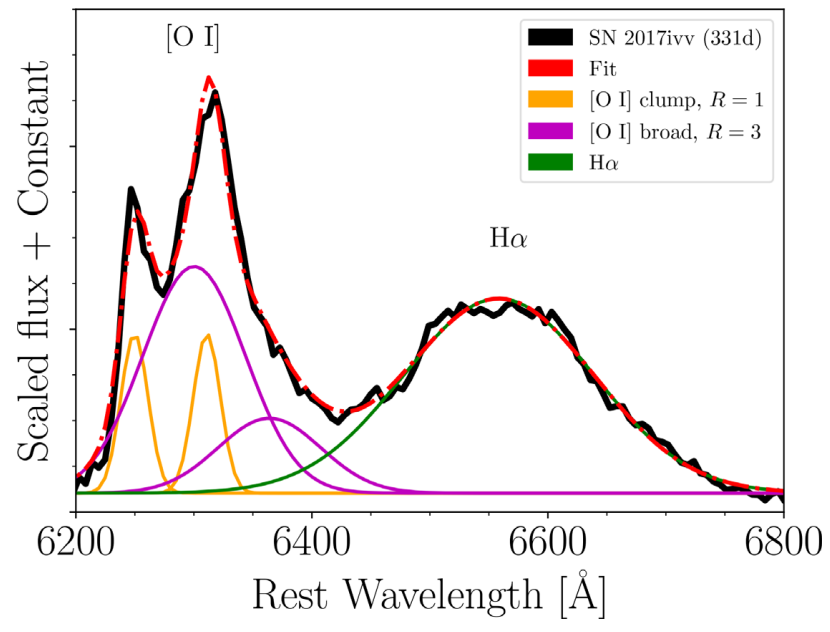

Figure 13. Best fit of the 6200-6800 A region of the spectrum of SN 2017 ivv at $331 \mathrm{~d}$. The observed spectrum is shown in black, the overall fit in red, the individual optically thin and optically thick components for [O I] in purple and orange, and the individual Gaussian profile for $\mathrm{H} \alpha$ in green.

with the same FWHM $\left(\sim 1300 \mathrm{~km} \mathrm{~s}^{-1}\right)$ and strength, separated by $64 \AA$, and with a blue-shift of $\sim 2400 \mathrm{~km} \mathrm{~s}^{-1}$ with respect to their rest-frame wavelengths (orange); two broad Gaussians centred at 6300 and 6364, which have the same FWHM $\left(\sim 4900 \mathrm{~km} \mathrm{~s}^{-1}\right)$ but the component at 6364 has a third the strength of the component at 6300; and a single (FWHM $\sim 8700 \mathrm{~km} \mathrm{~s}^{-1}$ ) Gaussian centred at $6563 \pm 10 \AA$ (green). Here, the blue-shifted and narrow profiles in [OI] could be interpreted as a pseudo-spherical distribution of the oxygen-rich ejecta with a localized clump travelling towards the observer with a projected blue-shifted velocity. As see in the figure, the bridge between $\mathrm{H} \alpha$ and [OI] can be reproduced by the [OI] $\lambda 6364$ component.

Trying to reproduce the nebular spectra of SN 1993J, SN 2008ax, and SN 2011dh, J15b found that [N II] $\lambda \lambda 6548,6583$ emission was more important than $\mathrm{H} \alpha$. In their models, the emission near $6550 \AA$ can be caused by $[\mathrm{N}$ II] $\lambda \lambda 6548,6583$ because the hydrogen envelopes in $\mathrm{SNe} \mathrm{IIb}$ are too small to produce any detectable $\mathrm{H} \alpha$ after $150 \mathrm{~d}$. At the same time, strong [N II] emission follows naturally from the quite massive $\mathrm{He} / \mathrm{N}$ shell $\left(\sim 0.5-1 \mathrm{M}_{\odot}\right)$, in which $\mathrm{N}$ is a much more efficient coolant than He. Fang \& Maeda (2018), analysing seven SNe IIb and two SN Ib, found support for this interpretation. Although the $[\mathrm{N}$ II] $\lambda \lambda 6548,6583$ is a possibility, we suggest the emission at $\sim 6550 \AA$ is related to $\mathrm{H} \alpha$. This suggestion is supported by the fact that $\mathrm{H} \alpha$ is stronger than the predicted [N II] emission. After $500 \mathrm{~d}$, the broad $\mathrm{H} \alpha$ emission indicates that the line arises from both the radioactive heating of hydrogen-rich ejecta from interior regions and the reverse shock interacting with high-velocity outer hydrogenrich material.

\subsubsection{Oxygen emission lines}

During the nebular phase, CC-SNe exhibit oxygen emission lines from O I $\lambda 7774$, [O I] $\lambda 5577$, and [O I] $\lambda \lambda 6300,6364$. The O I $\lambda 7774$ line is not detected in the spectra of SN 2017ivv. The [OI] $\lambda 5577$ and [OI] $\lambda \lambda 6300,6364$ lines become visible at the beginning of the nebular phase, but [O I] $\lambda 5577$ disappears more rapidly due to its higher temperature sensitivity. In SN 2017ivv, [O I] $\lambda 5577$ fades with time and is barely detected after $244 \mathrm{~d}$. The [O I] $\lambda \lambda 6300$, 6364 profile is detected during the nebular phase showing a double- 
peaked structure. The origin of the double-peaked [O I] $\lambda \lambda 6300$, 6364 emission line has been discussed by several authors (e.g. Milisavljevic et al. 2010, and references therein). A double peaked structure can be explained by a torus or disc-like geometry (e.g. Mazzali et al. 2001; Maeda et al. 2002; Mazzali et al. 2005; Maeda et al. 2006, 2008; Modjaz et al. 2008; Taubenberger et al. 2009). An alternative explanation, based on the separation of the two peaks ( $\sim 64 \AA$ ), suggests that the these peaks may originate from the two lines of the doublet from a single emitting source on the front of the SN ejecta which move towards the observer (Milisavljevic et al. 2010). Maurer et al. (2010) suggested that the double-peaked oxygen emission can also be caused by high-velocity (above $\sim 11000 \mathrm{~km} \mathrm{~s}^{-1}$ ) $\mathrm{H} \alpha$ absorption.

For SN 2017ivv, just the first scenario is fully plausible. Like $\mathrm{H} \alpha$, both [OI] $\lambda 5577$ and [OI] $\lambda \lambda 6300,6364$ show blue-shifted emission profiles over the entire nebular phase. For [O I] $\lambda 5577$, the blue-shift of the peak decreases from $\sim-2500 \mathrm{~km} \mathrm{~s}^{-1}$ at $110 \mathrm{~d}$ to $\sim-1900 \mathrm{~km} \mathrm{~s}^{-1}$ at $242 \mathrm{~d}$. The velocity offset of [O I] $\lambda \lambda 6300,6364$ line goes down from $\sim-1290 \mathrm{~km} \mathrm{~s}^{-1}$ at $111 \mathrm{~d}$ to $\sim-145 \mathrm{~km} \mathrm{~s}^{-1}$ at $520 \mathrm{~d}$ (the bottom panel of Fig. 10). As we see, these emission lines keep their blue-shifted velocities during all nebular observations. Similar behaviour was reported by Milisavljevic et al. (2010) for stripped-envelope SNe with double-peaked [OI] profiles. Multiple scenarios have been proposed to explain the blue-shift of [O I] in these objects, including ejecta geometry, contamination from other emission lines and opacity in the core of the ejecta (for more details see Taubenberger et al. 2009, and references therein). Based on the blue-shifts observed in all emission lines of SN 2017ivv, and their slow drop with time, the most consistent interpretation is asymmetries in the ejecta (geometric scenario; see Section 6.7). In the early nebular phases, indications of additional blue-shifting, likely due to residual atomic opacity, are also seen.

On the left panel of Fig. 12, we show the evolution of the doublepeaked [OI] $\lambda \lambda 6300,6364$ profile of SN 2017ivv. The [OI] line exhibits a quite unusual evolution. Between 110 and $158 \mathrm{~d}$, [O I] shows two emission peaks that resemble the nebular profiles of SN II. During this period, the blue and red peaks have equal strengths, expected in the optically thick limit (Jerkstrand et al. 2014). In the optically thin limit, [O I] $\lambda 6364$ is always three times weaker than [O I] $\lambda 6300$ (e.g. Spyromilio \& Pinto 1991; Li \& McCray 1992; Taubenberger et al. 2009; Jerkstrand et al. 2014), but in SN 2017ivv, from 242 to $337 \mathrm{~d}$, the redder peak gradually becomes stronger than the bluer one. Some stripped-envelope SNe (e.g. SN 1996aq Valenti et al. 2008; Taubenberger et al. 2009 and SN 2003jd; Mazzali et al. 2005; Valenti et al. 2008) have shown this behaviour. In Fig. 14, we compare SN 2017ivv at 278 d with one of these objects, SN 1996aq (Taubenberger et al. 2009) at $268 \mathrm{~d}$. The [O I] profile in both objects is quite similar: identical strengths in the red and blue peaks together with comparable line width.

Maeda et al. (2002) showed that the emission profiles in SNe Ic can be affected by an aspherical explosion, and the double-peaked profiles can be produced when the degree of asymmetry is high. Similar results were also found by Mazzali et al. (2005). Using an aspherical explosion model, they found that the double-peaked profile of the [O I] line in SN 2003jd can be reproduced by a jet axis oriented $>70^{\circ}$ away from the line of sight. Asymmetry with respect to the equatorial plane of the ejecta are shown to be able to give different strengths of the two peaks; for fixed viewing angle with respect to $z$ (the axis of the bipolar explosion asymmetry), the red or blue peak can be stronger depending on whether angle is with respect to $+z$ or $-z$. Therefore, a geometric scenario fully supports the blue-shifted emission lines and the double-peaked structure in [O I].

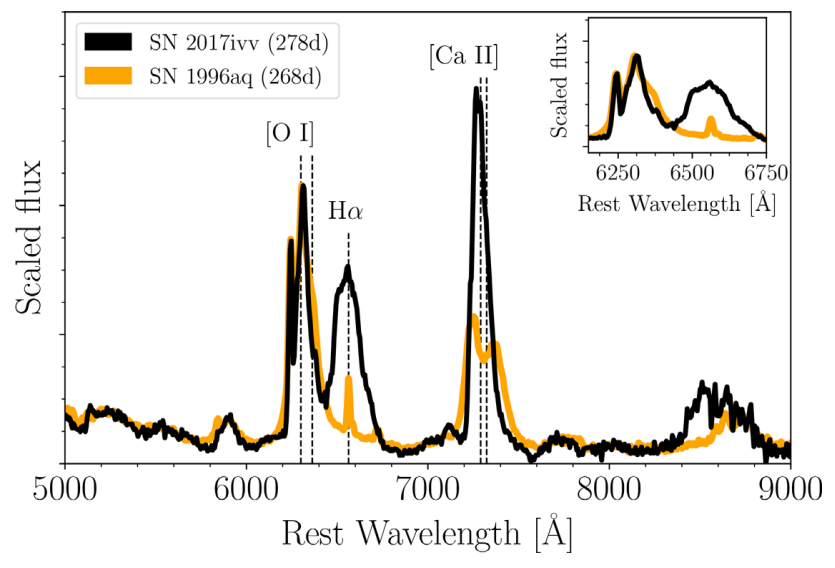

Figure 14. Spectral comparison of SN 2017ivv at $278 \mathrm{~d}$ with the Type Ib SN 1996aq at $268 \mathrm{~d}$. Spectra are scaled to match the [O I] $\lambda \lambda 6300,6364$ doublet.

While the two peaks of [O I] separated by $\sim 63-68 \AA$ (Fig. B1 in the online supplementary material) support the doublet nature of the [O I ] line emission, the structure of the [O I] profile could exclude this possibility. This is because the asymmetry of the lines (the redder peak stronger than the blue peak) can be simply explained by an aspherical explosion. Finally, we rule out the latter scenario (highvelocity $\mathrm{H} \alpha$ absorption) based on the Gaussian analysis performed in Section 6.2.1 (Fig. 13).

After $506 \mathrm{~d}$, the two emission peaks of [OI] $\lambda \lambda 6300,6364$ show again equal strength, resembling the first three spectra of the nebular phase. The decreasing[O I] intensity can be an effect of CSM interactions.

\subsubsection{Calcium emission lines}

The evolution of the $[\mathrm{CaII}] \lambda \lambda 7291,7324$ emission-line profile is shown in the right-hnad panel of Fig. 12. During the nebular observations, the strength of [Ca II] changes significantly. From 110 and $158 \mathrm{~d}$, [Ca II] slowly evolves, with an equivalent width (EW) from $161 \AA$ increasing to $418 \AA$, and by $242 \mathrm{~d}$ it goes up to $\sim 1200 \AA$. At $506 \mathrm{~d}$, the pEW of the line decreases considerably to $\sim 150 \AA$. The decrease on the strength of the lines after $500 \mathrm{~d}$ could again be related to CSM interaction.

A double-peaked structure is detected in the top of the [Ca II] line between 110 and 337 d, becoming more evident from 242 to $337 \mathrm{~d}$. In addition to this double-peaked structure, [Ca II] shows a red shoulder which could be attributed to [Ni II] $\lambda 7378$ (Jerkstrand et al. 2015a). In terms of velocity, both the FWHM velocity and velocity offset (Fig. 10) show little evolution. The FWHM velocity is $\sim 4000 \mathrm{~km} \mathrm{~s}^{-1}$, which lies in the observed range of both SN II (Maguire et al. 2010; Silverman et al. 2017) and SNe IIb.

\subsection{Temperature estimation from the ratio of the oxygen lines}

The ejecta temperature at a given time can be constrained by the ratio of [O I] $\lambda 5577$ to [O I] $\lambda \lambda 6300,6364$ (e.g. Houck \& Fransson 1996; Jerkstrand et al. 2014). Both [O I] lines are excited by thermal collisions, but as they have different excitation energies, their line ratio depends on the temperature (e.g. Fransson \& Chevalier 1989; Jerkstrand et al. 2014). The [OI] $\lambda 5577$ line starts deviating from local thermodynamic equilibrium (LTE) after $\sim 250 \mathrm{~d}$ in SNe II and after $\sim 150 \mathrm{~d}$ in SNe IIb (Jerkstrand et al. 2014; J15b). At too early 
Table 1. Local thermodynamic equilibrium (LTE) temperatures and O I masses estimated by using the [O I] $\lambda 5577$ to [O I] $\lambda \lambda 6300,6364$ ratio.

\begin{tabular}{|c|c|c|c|c|c|c|c|c|}
\hline $\begin{array}{l}\text { Time } \\
\text { (d) } \\
\text { (1) }\end{array}$ & $\begin{array}{c}L_{5577} \\
\left(10^{38}\right) \mathrm{erg} \mathrm{s}^{-1} \\
(2)\end{array}$ & $\begin{array}{c}L_{6300,6364} \\
\left(10^{38}\right) \mathrm{erg} \mathrm{s}^{-1} \\
\text { (3) }\end{array}$ & $\begin{array}{l}T_{\beta_{\text {ratio }}=1} \\
\quad(\mathrm{~K}) \\
(4)\end{array}$ & $\begin{array}{c}T_{\beta_{\text {ratio }}=1.5} \\
(\mathrm{~K}) \\
(5)\end{array}$ & $\begin{array}{l}T_{\beta_{\text {ratio }}=2} \\
\quad(\mathrm{~K}) \\
(6)\end{array}$ & $\begin{array}{c}M(\mathrm{OI})_{\beta_{\text {ratio=1 }}^{*}} \\
\left(\mathrm{M}_{\odot}\right) \\
(7)\end{array}$ & $\begin{array}{c}M(\mathrm{O} \mathrm{I})_{\beta_{\text {ratio=1.5 }}^{*}} \\
\left(\mathrm{M}_{\odot}\right) \\
(8)\end{array}$ & $\begin{array}{c}M(\mathrm{O} \mathrm{I})_{\beta_{\text {ratio=2 }}^{*}} \\
\left(\mathrm{M}_{\odot}\right) \\
(9)\end{array}$ \\
\hline 136 & 7.35 & 26.71 & 5233 & 4836 & 4588 & 0.42 & 0.60 & 0.78 \\
\hline 158 & 6.04 & 30.90 & 4894 & 4544 & 4325 & 0.66 & 0.94 & 1.22 \\
\hline 242 & 1.04 & 15.22 & 4078 & 3832 & 3675 & 0.82 & 1.18 & 1.52 \\
\hline
\end{tabular}

Notes. Columns: (1) time from explosion; (2) line luminosity of [O I] $\lambda 5577$; (3) line luminosity of [O I] $\lambda \lambda 6300,6364$; (4) temperature assuming $\beta_{\text {ratio }}=1$;

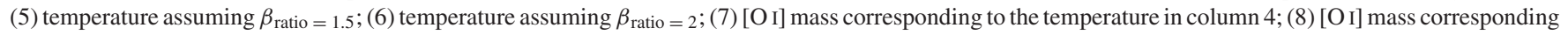
to the temperature in column 5 ; (9) [O I] mass corresponding to the temperature in column 6.

${ }^{*}$ Assuming $\beta_{6300,6364}=0.5$.

phases $(\lesssim 100 \mathrm{~d})$ radiative transfer and line blending effects become problematic. An LTE line ratio analysis is therefore most suitable at $\sim 150-200$ d. As shown in Fig. 7, [O I] $\lambda 5577$ is clearly detected in our first four nebular spectra, from 110 to $242 \mathrm{~d}$.

The temperature given a certain line ratio of the [OI] lines, assuming LTE, is given by the following equation

$$
\frac{L_{5577}}{L_{6300,6364}}=38 \times \exp \left(\frac{-25790 K}{T}\right) \frac{\beta_{5577}}{\beta_{6300,6364}},
$$

where the $\beta_{5577} / \beta_{6300,6364}$ ratio ( $\left.\beta_{\text {ratio }}\right)$ must be in the range of $1-2$ if partial transitioning to optical thinness for [O I] $\lambda \lambda 6300,6364$ is either observed or can be inferred from any reasonable values of oxygen mass given the epoch and expansion velocities (Jerkstrand et al. 2014). Assuming $\beta_{5577} / \beta_{6300,6364}=1,1.5$ and 2 , we find the temperatures listed Table 1 (columns 4,5 and 6, respectively). In this table, we also report the measured line luminosities of [O I] $\lambda 5577$ and [O I] $\lambda \lambda 6300,6364$, and the estimated oxygen mass corresponding to each temperature (see Section 6.4). Looking at the temperatures obtained for SN 2017ivv at around 158 days with those calculated for SN 1993J, SN 2008ax, and SN 2011dh at similar epochs (J15b), we find that SN 2017ivv has a temperature comparable with these Type IIb events. Unfortunately, [O I] $\lambda 5577$ is not clearly detected in the majority of late spectra of SNe II, but some weak detections have been made before (e.g. SN 2012aw; Jerkstrand et al. 2014), although at later phases.

\subsection{Oxygen mass}

Using the temperature estimated in Section 6.3, the O I mass can be derived from the oxygen luminosity as follows:

$$
M_{\mathrm{O}}=\frac{L_{6300,6364} / \beta_{6300,6364}}{9.7 \times 10^{41} \mathrm{erg} \mathrm{s}^{-1}} \times \exp \left(\frac{22720 \mathrm{~K}}{T}\right) \mathrm{M}_{\odot}
$$

(Jerkstrand et al. 2014). Taking $\beta_{6300,6364}=0.5$, we obtain the O I masses presented in columns 7,8 and 9 of Table 1. Comparing the value at $\sim 158$ days to those obtained for the SN IIb 1993J, $\mathrm{SN} 2008 \mathrm{ax}$, and SN 2011dh $\left(M_{\mathrm{O}} \sim 0.3-0.75 \mathrm{M}_{\odot}\right.$, for $\beta_{\text {ratio }}=$ 1 and $\left.\beta_{6300,6364}=0.5 ; \mathrm{J} 15 \mathrm{~b}\right)$, we note that SN 2017ivv has an O I mass in the SN IIb range (column 7, Table 1). Unfortunately, fastdeclining SNe II do not have a mass estimation using this method. For $\beta_{6300,6364}=1$ (i.e. assuming optically thin emission), we can obtain the minimum mass of oxygen required to produce the observed [OI]. With this assumption, the derived [OI] masses are half of those presented in Table 1. We note that these oxygen masses are comparable to those obtained using the relation presented by Uomoto (1986).

From Table 1, we also note a large range in the [O I] masses. $\mathrm{J} 15 \mathrm{~b}$ discuss how time-dependent radiative transfer effects and/or deviations from LTE can lead to different results at different epochs.
For IIb SNe, the optimum time to estimate the [O I] mass is around 150 days; before this time, the lines can be affected by radiative transfer effects, and at later times, the $[\mathrm{O}$ I $] \lambda 5577$ line starts to deviate from LTE. For SN 2017ivv, at $158 \mathrm{~d}$, we found a $M(\mathrm{O} \mathrm{I})=0.66 \mathrm{M}_{\odot}$ for $\beta_{\text {ratio }=1}, \mathrm{M}(\mathrm{OI})=0.94 \mathrm{M}_{\odot}$ for $\beta_{\text {ratio }=1.5}$ and $\mathrm{M}(\mathrm{OI})=1.22 \mathrm{M}_{\odot}$ for $\beta_{\text {ratio }=2}$, these values correspond to a $15-19 \mathrm{M}_{\odot}$ progenitor (Jerkstrand et al. 2014).

\section{$6.5[\mathrm{Ca} \mathrm{II}] /[\mathrm{O} \mathrm{I}]$ ratio}

Fransson \& Chevalier (1989) showed that the relative abundances of the different elements vary substantially with the mass of the helium core. Specifically, the $[\mathrm{Ca} I \mathrm{II}] /[\mathrm{OI}]$ ratio can be used to estimate the ZAMS mass of the progenitor. Several authors (e.g. Fransson \& Chevalier 1989; Elmhamdi et al. 2004; Kuncarayakti et al. 2015) have used this ratio as a diagnostic of the core mass. However, some issues emerge from these assumptions. Li \& McCray (1993) demonstrated that in $\mathrm{H}$-rich $\mathrm{SNe}$ most of the emission tends to come from primordial calcium in the hydrogen envelope. In $\mathrm{SNe} \mathrm{IIb}$, the $\mathrm{H}$ envelopes are too small for such a contribution, and the $\mathrm{Ca}$ emission comes from the explosive oxygen burning zone. The mass of this zone depends on the explosion energy (Jerkstrand 2017), and therefore the link between $[\mathrm{Ca} I I] /[\mathrm{OI}]$ ratio and the core mass is complex.

Taking into account these caveats, we estimate the flux of these emission lines of SN 2017ivv and their ratios in Fig. 15. From the top panel, one sees that all lines in SN 2017ivv evolve with time. The most extreme evolution is seen in the $\mathrm{H} \alpha$ line; $[\mathrm{Ca}$ II $]$ and [OI] evolve more slowly. Their ratio, shown in the bottom panel of Fig. 15, decreases from $1.76 \pm 0.17$ at $110 \mathrm{~d}$ to $0.55 \pm 0.15$ at $671 \mathrm{~d}$. Between 110 and $158 \mathrm{~d}$, the ratio is larger than 1.5, which is consistent with the values presented for SNe II by Kuncarayakti et al. (2015), while after $242 \mathrm{~d}$ the values are smaller and comparable to those of stripped envelope SNe (Kuncarayakti et al. 2015). In Fig. 16, we compare the line ratio for SN 2017 ivv with that of other CC-SNe. The ratio at 285 and 331 days is highlighted with darker contours showing similar age than the comparison sample. Although the ratio differs by $\sim 0.2$, they are still consistent with the values of the stripped envelope SNe. The ratio of the slow-declining SN II 2015bs also lies in this region, but at later epoch than other comparison objects.

The evolution of the $[\mathrm{CaII}] /[\mathrm{OI}]$ ratio in SN 2017ivv carries problems for any estimate of mass of the helium core: if we take the ratio at earlier phases (before $200 \mathrm{~d}$ ), we are led to conclude that the $\mathrm{SN}$ has a low helium core mass (larger ratio, smaller masses). However, if we consider the ratio after 200-300 d, the result implies a more massive helium core. Valenti et al. (2014) and Terreran 

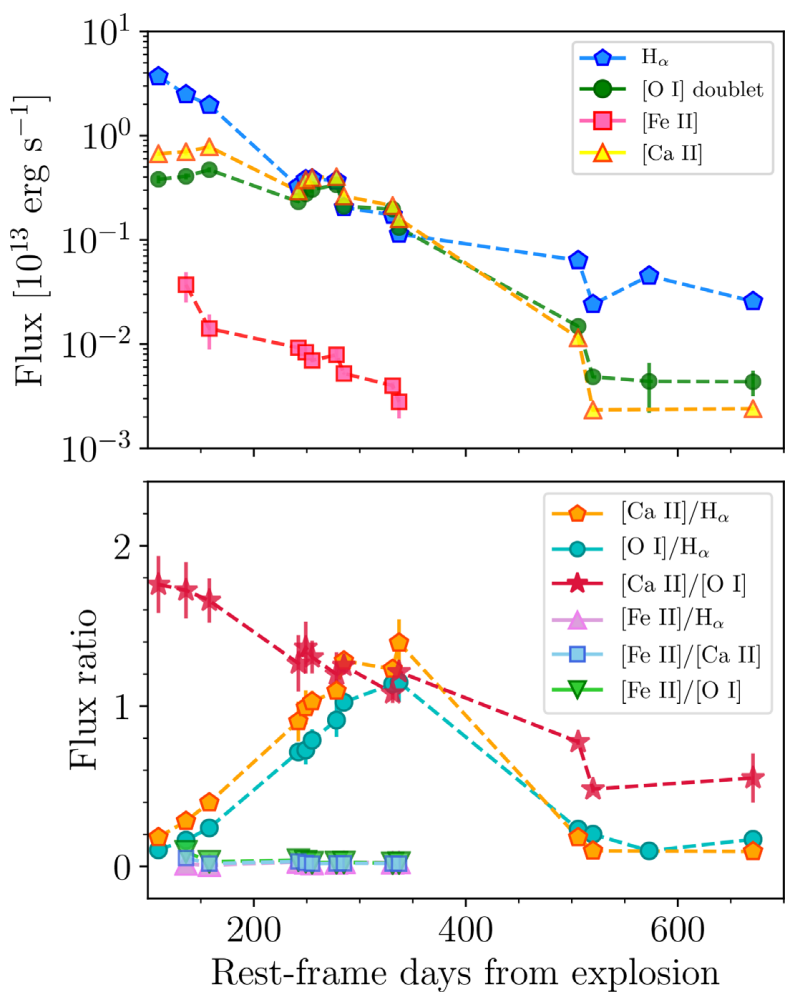

Figure 15. Top panel: Flux evolution of $\mathrm{H} \alpha$, [O I], [Ca II], and [Fe II] in SN 2017ivv. Bottom panel: Flux ratio of the emission lines in SN 2017ivv.

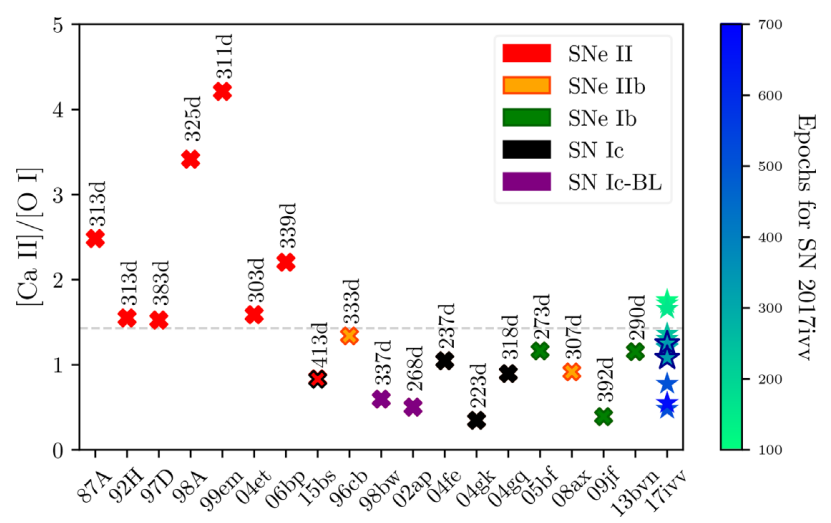

Figure 16. $[\mathrm{Ca} I \mathrm{II}] /[\mathrm{OI}]$ line ratio for several $\mathrm{CC}-\mathrm{SNe}$ at similar ages during the nebular phase. The SN phases are computed with respect to the time of maximum light. Red crosses indicate SN II, orange SN IIb, green SN Ib, black SN Ic, and purple broad-lined Type-Ic. SN 2017ivv is shown with star symbols. SN 2017ivv is colour-coded respect to the epoch from the explosion. Stars with darker contours show similar age than the comparison sample. The horizontal dashed line indicates the limit between SNe II and stripped envelope events $(\sim 1.43)$. Figure adapted from Kuncarayakti et al. (2015).

et al. (2019), using a large sample of CC-SNe, found that this line ratio generally evolves with time, and Jerkstrand (2017) show that stripped-envelope models typically predict a declining ratio with time. Using their fig. 13, the observed ratio of $\sim 1$ at $300 \mathrm{~d}$ most closely matches 3-4 $\mathrm{M}_{\odot}$ He core models, which would correspond to a $M_{\text {ZAMS }} \lesssim 15 \mathrm{M}_{\odot}$ progenitor (if its a SN IIb). However, as they discuss, the ratio is sensitive to various parameters (e.g. explosion energy, mixing, and composition details).

\subsection{Blue-shifted lines}

We investigate whether opacity effects can produce the observed asymmetric lines profiles in SN 2017ivv by modelling a uniform sphere with a velocity of $3500 \mathrm{~km} \mathrm{~s}^{-1}$, with the optical depth $\tau$ and destruction probability $\epsilon$ as the two parameters. Starting with models having pure scattering $(\epsilon=0)$, no choice of optical depth produces the right combination of a blue-shifted peak and red wing for [Ca II] (Fig. 17, top panel). Although scattering leads to a blue-shifting of the peak, it is not sufficient and the red-wing is overproduced.

Moving on to explore models where (partial) destruction is allowed for, we find that for $\epsilon \sim 0.5$ (50 percent destruction, 50 percent scattering), the line profiles come into quite good agreement (Fig. 17, bottom panel). In this model, some scattering is still needed to make the 'red tail' of the lines, which extends up to about $6000 \mathrm{~km} \mathrm{~s}^{-1}$, while absorption is needed to push the peak sufficiently to the blue (absorption shifts the peak more strongly than scattering).

This exercise demonstrated that the asymmetric line profiles can in principle be produced by radiative transfer effects, even in a simple toy model, and thus the necessity of an asymmetric ejecta cannot be directly established without more detailed modelling. The most plausible candidate for this opacity is line blocking which has been demonstrated to operate in stripped-envelope SNe up to $\sim 200 \mathrm{~d}$ (J15b). Depending on whether absorbed photons resonance scatter or fluoresce (true thermalization is unimportant), various epsilon values can be conceived of and a value of around 0.5 as indicated here is plausible. The main challenge for this mechanism is to sustain significant opacity for long enough. In the J15b models the optical depths became too small to significantly affect the lines profiles after about $200 \mathrm{~d}$. That also corresponds to the epoch around which line peak blue-shifts evolution ceased in many $\mathrm{Ib} / \mathrm{IIb} \mathrm{SNe}$.

Current 3D SN explosion simulations (e.g. Wongwathanarat, Janka \& Müller 2013; Wongwathanarat, Müller \& Janka 2015) predict asymmetries in line profiles with line centroid shifts up to about $\pm 500 \mathrm{~km} \mathrm{~s}^{-1}$ for the IIP case (Jerkstrand et al. 2020). For stripped envelope $\mathrm{SNe}$, the scales of these shifts are likely yet larger due to overall higher velocities. Thus, it would not be surprising to see lines shifted by order $\pm 1000 \mathrm{~km} \mathrm{~s}^{-1}$ in a stripped-envelope SN. SN 1993J and SN 2008ax settled on line peak shifts of about $-500 \mathrm{~km} \mathrm{~s}^{-1}$ (blue-shifts), whereas SN 2011dh settled on almost symmetric or slightly red-shifted lines (Ergon et al. 2014, 2015). The settling of $2017 \mathrm{ivv}$ on $1000-2000 \mathrm{~km} \mathrm{~s}^{-1}$ blue-shifts is thus quite remarkable, but it is perhaps not out of the ballpark of what current standard explosion models predict for a viewing angle close to the bulk momentum vector of the ejecta.

\section{7 $\mathrm{SN}$ progenitor}

After $500 \mathrm{~d}$, the spectra and light curves of SN 2017ivv show significant signs of interaction between the SN ejecta and the CSM. These signs suggest that the SN progenitor must have lost a large amount of its hydrogen envelope before the explosion. Given that SN 2017ivv exploded in a low-metallicity environment, several considerations must be taken. If we assume a relation between metallicity and mass-loss (Heger et al. 2003), at low-metallicity the mass-loss through winds should be less efficient. This may make the binary progenitor scenario more likely. On the other hand, if we consider the mass-loss of single RSGs appears to be independent of metallicity (van Loon et al. 2005; Goldman et al. 2017; Chun et al. 2018; Gutiérrez et al. 2018), the metallicity-driven winds are still possible. However, a more massive progenitor $\left(>20 \mathrm{M}_{\odot}\right)$ is maybe needed (Claeys et al. 2011, and references therein). 

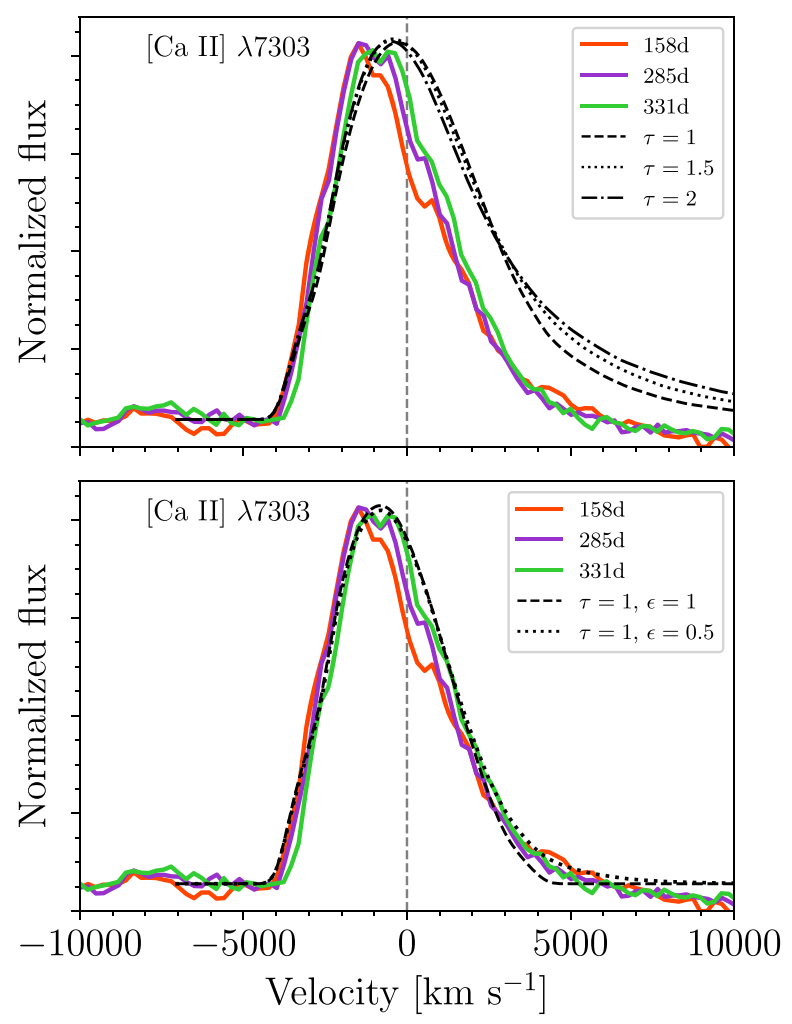

Figure 17. Top panel: Observed [Ca II] line at 158, 285, and $331 \mathrm{~d}$ compared to models of a uniform sphere with different degree of scattering optical depth. Bottom panel: Observed [Ca II] line at 158, 285, and $331 \mathrm{~d}$ compared with a fully destructive $(\epsilon=1)$ and partially destructive $(\epsilon=0.5)$ opacity model. The zero velocity of [Ca II] is at $7303 \AA$. The models are normalized to match the peak flux.

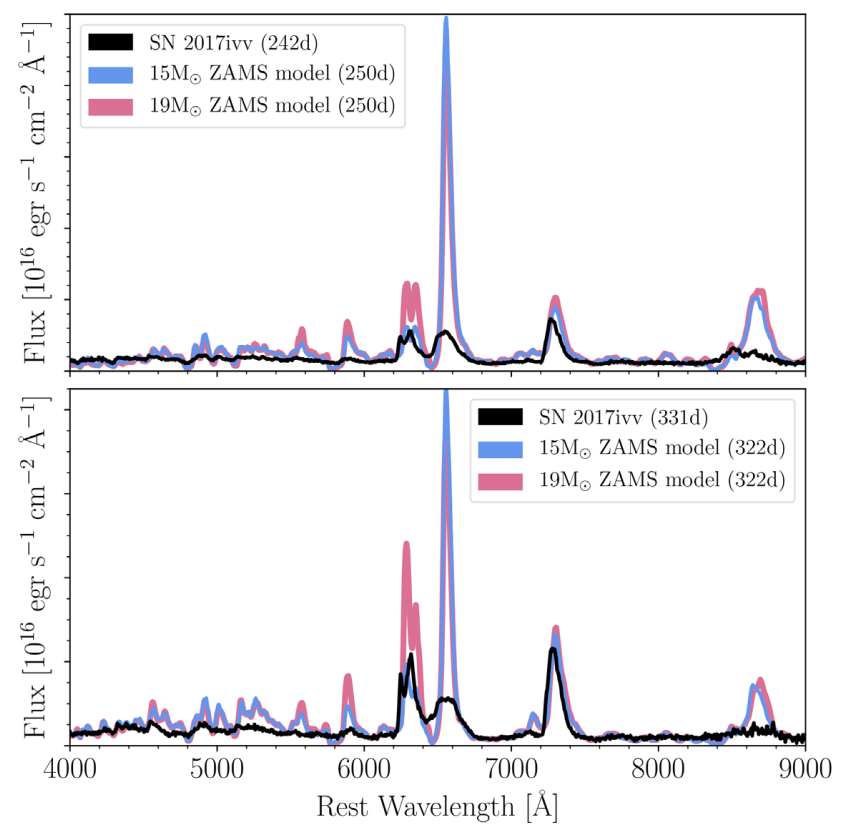

We tried to fit the light curve using hydrodynamical simulations (Limongi \& Chieffi, in preparartion) starting from 'realistic' pre-SN models (Limongi \& Chieffi 2018) by changing the explosion energy. None of these simulations were able to reproduce the observations. The only way to fit the data was by artificially reducing the ejecta mass to values as low as $\sim 0.8 \mathrm{M}_{\odot}$. This confirms that the binary scenario is the more likely.

To constrain the progenitor mass, we use the late-time spectral models of Jerkstrand et al. (2014) and J15b. We compare two spectra of SN 2017ivv, at 242 and $331 \mathrm{~d}$ with the 15 and $19 \mathrm{M}_{\odot}$ models for SNe II (Jerkstrand et al. 2014), and 13 and $17 \mathrm{M}_{\odot}$ models for SNe IIb (J15b). As the strength of the [O I] $\lambda \lambda 6300,6364$ shows a correlation with the progenitor ZAMS mass (Jerkstrand et al. 2012, 2014), a match with the $[\mathrm{O} I]$ lines will provide good constraints. To scale the models, we used the relation (2) from Bostroem et al. (2019):

$\frac{F_{\mathrm{obs}}}{F_{\mathrm{mod}}}=\frac{d_{\mathrm{mod}}^{2}}{d_{\mathrm{obs}}^{2}} \frac{M\left({ }^{56} \mathrm{Ni}\right)_{\mathrm{obs}}}{M\left({ }^{56} \mathrm{Ni}\right)_{\mathrm{mod}}} \exp \left(\frac{t_{\mathrm{mod}}-t_{\mathrm{obs}}}{114.4}\right)$,

where $F_{\text {obs }}$ and $F_{\text {mod }}$ are the observed and models fluxes, $d_{\text {obs }}^{2}$ and $d_{\text {mod }}^{2}$ are the observed and model distances, $M\left({ }^{56} \mathrm{Ni}\right)_{\text {obs }}$ and $M\left({ }^{56} \mathrm{Ni}\right)_{\bmod }$, the observed and model ${ }^{56} \mathrm{Ni}$ masses, and $t_{\mathrm{obs}}$ and $t_{\text {mod }}$ are the phases of the observation and the model. The comparison is presented in Fig. 18. At $242 \mathrm{~d}$, we find that the [O I] strength matches with the $15 \mathrm{M}_{\odot}$ model for SNe II, while at $331 \mathrm{~d}$, the [OI] strength falls between the 15 and $19 \mathrm{M}_{\odot}$. However, the severe discrepancy with the $\mathrm{H} \alpha$ fluxes makes it difficult to put much weight on these numbers. In comparisons with SN IIb models, the discrepancy goes the other way (SN 2017ivv likely has more $\mathrm{H}$ than these models) but is arguably less severe. We find that at $242 \mathrm{~d}$, [O I] strength falls between the 13 and $17 \mathrm{M}_{\odot}$ model, but at $331 \mathrm{~d}$, the strength of [OI] is larger than the $17 \mathrm{M}_{\odot}$ model. The IIb models have a too rapidly increasing gamma-ray escape compared to SN 2017ivv, as clarified by them being significantly too dim at $330 \mathrm{~d}$. Thus, the $240 \mathrm{~d}$ comparison (where the trapping is more in agreement) is more relevant than the $330 \mathrm{~d}$ one. Given that the [O I] $\lambda \lambda 6300,6364$ doublet shows an

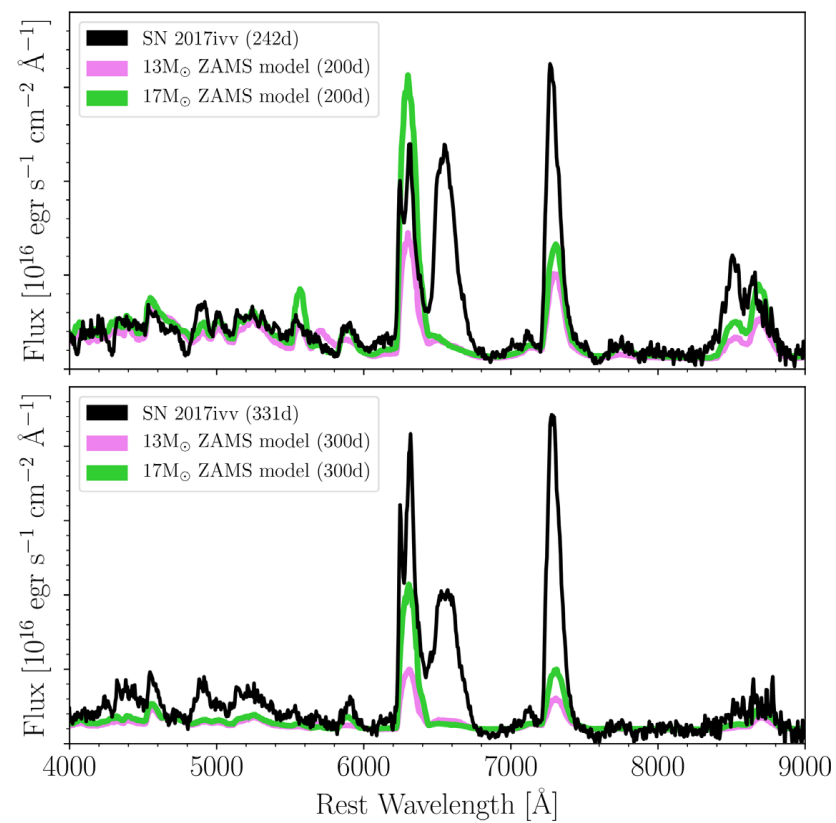

Figure 18. Comparison of the nebular spectra of SN 2017ivv at $331 \mathrm{~d}$ with the nebular models. Left-hand panel: Comparison with SN II models of $M_{\mathrm{ZAMS}}$ of 15 and $19 \mathrm{M}_{\odot}$ (Jerkstrand et al. 2014). Right-hand panel: Comparison with a SN IIb model of $M_{\text {ZAMS }}=13$ (13G) and $17 \mathrm{M}_{\odot}$. The models were scaled to same distance and same ${ }^{56} \mathrm{Ni}$ mass as SN 2017 ivv. 
unusual evolution in SN 2017ivv, the constraints on the progenitor mass must be analysed with caution. However, based on the spectral comparison, we estimate a progenitor between 15 and $17 \mathrm{M}_{\odot}$.

\section{SUMMARY AND CONCLUSIONS}

We have presented the photometric and spectroscopic observations of the core collapse supernova, SN 2017ivv. SN 2017ivv exploded in the faintest host galaxy $\left(M_{r}=-10.3 \mathrm{mag}\right)$ reported for an SN II to date. Analysing the observed properties at early and late times, we found that SN 2017ivv shares properties with the fast-declining SN II and SN IIb. Based on the unusual evolution and the emission lines during the nebular phase, we suggest that SN 2017ivv has a lower hydrogen mass than a typical SN II but more than a SN IIb.

At early times, the light curve of SN 2017ivv shows a fast rise (6-8 d) to the peak $\left(M_{g}^{\max }=-17.84 \mathrm{mag}\right)$, followed by a very rapid decline $(7.94 \pm 0.48 \mathrm{mag}$ per $100 \mathrm{~d}$ in the $V$ band). The spectra show broad P-Cygni profiles, with $\mathrm{H} \alpha$ exhibiting an asymmetric emission component and a weak absorption feature. At late times, based on the spectroscopic behaviour, we could split the evolution into three phases: (1) $\mathrm{H} \alpha$ strong phase ( $<200 \mathrm{~d})$; (2) $\mathrm{H} \alpha$ weak phase (between 200 and $350 \mathrm{~d}$ ); and (3) $\mathrm{H} \alpha$ broad phase (>500 d). In phase one, SN 2017ivv shows the typical spectra of a SN II: a dominant $\mathrm{H} \alpha$ emission profile, and clear detections of the [O I] and [Ca II] lines. In phase two, $\mathrm{H} \alpha$ develops a boxy emission profile, while [OI] and [CaII] become the strongest lines in the spectra. The $[\mathrm{O}$ I] $\lambda \lambda 6300,6364$ displays an unusual profile with the red peak stronger than the blue one. These properties, observed before in some stripped-envelope $\mathrm{SNe}$, can be associated with aspherical explosions. Finally, in phase three, the spectra display weak [O I] and [Ca II] lines, combined with a broad and strong $\mathrm{H} \alpha$ emission. These characteristics are likely explained by CSM interaction, which is also supported by the flattening in the light curve at late-times.

Taking into account all these properties, we concluded that SN 2017ivv arises from an asymmetric explosion and that its progenitor had a significant mass-loss prior to the explosion. As it explodes in a low-metallicity environment, a binary progenitor system is the most likely scenario. From the nebular analysis, we infer a progenitor mass of $15-17 \mathrm{M}_{\odot}$.

\section{ACKNOWLEDGEMENTS}

We thank the anonymous referee for the comments and suggestions that have helped to improve the paper.

We are grateful to Marco Limongi and Giacomo Terreran for useful discussion. We thank Richard S. Post its contribution with data from the Post Observatory.

CPG and MS acknowledge support from EU/FP7-ERC grant no. 615929. AJ acknowledges funding by ERC Starting grant no. 803189 and Swedish National Research Council grant no. 2018-03799. LG was funded by the European Union's Horizon 2020 research and innovation programme under the Marie Skłodowska-Curie grant agreement no. 839090. MF is supported by a Royal Society - Science Foundation Ireland University Research Fellowship. KM acknowledges support from ERC Starting grant no. 758638. SJS acknowledges funding from STFC grant no. ST/P000312/1. TMB was funded by the CONICYT PFCHA/DOCTORADOBECAS CHILE/2017-72180113. TWC acknowledges EU Funding under Marie Skłodowska-Curie grant agreement no. $842471 \mathrm{MG}$ is supported by the Polish NCN MAESTRO grant no. 2014/14/A/ST9/00121. DAH and GH were supported by NSF grant no. AST-1313484. MN is supported by a Royal
Astronomical Society Research Fellowship. Support for GP and JLP is provided by the Ministry of Economy, Development, and Tourism's Millennium Science Initiative through grant no. IC120009, awarded to The Millennium Institute of Astrophysics, MAS. JLP is also supported by FONDECYT through the grant no. 1191038. Research by KAB and SV is supported by NSF grant no. AST-1813176. CSK is supported by NSF grants AST-1515927 and AST-181440. CSK and BJS are supported by NSF grant no. AST-1907570. BJS is also supported by NSF grants AST-1920392 and AST-1911074. JB, DH, and DAH are supported by NSF AST-1911225.

This work used data collected at the European Organisation for Astronomical Research in the Southern Hemisphere, Chile, under programme IDs: 0104.D-0503(A), 0103.D-0393(A), 0103.D0440(A), 0102.D-0356(A), 0102.A-9099(A), 0101.A-9099(A), and as part of PESSTO (the Public ESO Spectroscopic Survey for Transient Objects Survey) ESO programme 1103.D-0328 and 199.D0143.

This work makes use of data from Las Cumbres Observatory telescope network.

Part of the funding for GROND (both hardware as well as personnel) was generously granted from the Leibniz-Prize to Prof. G. Hasinger (DFG grant no. HA 1850/28-1).

This research uses data obtained through the Telescope Access Program (TAP), which has been funded by the National Astronomical Observatories of China, the Chinese Academy of Sciences, and the Special Fund for Astronomy from the Ministry of Finance.

Partially based on observations collected at Copernico $1.82 \mathrm{~m}$ and Schmidt 67/92 telescopes (Asiago, Italy) of the INAF - Osservatorio Astronomico di Padova.

The Liverpool Telescope is operated on the island of La Palma by Liverpool John Moores University in the Spanish Observatorio del Roque de los Muchachos of the Instituto de Astrofisica de Canarias with financial support from the UK Science and Technology Facilities Council.

Some of the data presented herein were obtained at the W. M. Keck Observatory, which is operated as a scientific partnership among the California Institute of Technology, the University of California and the National Aeronautics and Space Administration. The Observatory was made possible by the generous financial support of the W. M. Keck Foundation.

We thank the Las Cumbres Observatory and its staff for its continuing support of the ASAS-SN project. ASAS-SN is supported by the Gordon and Betty Moore Foundation through grant no. GBMF5490 to the Ohio State University, and NSF grants AST-1515927 and AST1908570. Development of ASAS-SN has been supported by NSF grant no. AST-0908816, the Mt. Cuba Astronomical Foundation, the Center for Cosmology and AstroParticle Physics at the Ohio State University, the Chinese Academy of Sciences South America Center for Astronomy (CAS- SACA), the Villum Foundation, and George Skestos.

This work has made use of data from the Asteroid Terrestrialimpact Last Alert System (ATLAS) project. ATLAS is primarily funded to search for near earth asteroids through NASA grants NN12AR55G, 80NSSC18K0284, and 80NSSC18K1575; by products of the NEO search include images and catalogues from the survey area. The ATLAS science products have been made possible through the contributions of the University of Hawaii Institute for Astronomy, the Queen's University Belfast, the Space Telescope Science Institute, and the South African Astronomical Observatory.

This work has been partially supported by the Spanish grant PGC2018-095317-B-C21 within the European Funds for Regional Development (FEDER). 
This research has made use of the NASA/IPAC Extragalactic Database (NED) which is operated by the Jet Propulsion Laboratory, California Institute of Technology, under contract with the National Aeronautics. We acknowledge the usage of the HyperLeda data base (http://leda.univ-lyon1.fr).

Facilities: NTT (EFOSC2); VLT (MUSE, FORS2); Las Cumbres Observatory; ATLAS; Keck: I (LRIS); 1.82 m Copernico (AFOSC); 67/91 Schmit Telescope; 1.22 Galileo (BC); ASAS-SN; Liverpool Telescope (SPRAT); Tillinghast (FAST); 2.2-m MPG telescope (GROND); Post Observatory SRO

Software: PYTHON from https://www.python.org/; IRAF, ESOREFLEX pipeline (Freudling et al. 2013); LPIPE (Perley 2019); GROND pipeline (Krühler et al. 2008), SNID (Blondin \& Tonry 2007), GELATO (Harutyunyan et al. 2008), CIGALE (Boquien et al. 2019).

\section{DATA AVAILABILITY}

The data underlying this article are available in the supporting information section (Appendix A) and through the WISeREP (https: //wiserep.weizmann.ac.il/home) archive (Yaron \& Gal-Yam 2012).

\section{REFERENCES}

Alard C., 2000, A\&AS, 144, 363

Alard C., Lupton R. H., 1998, ApJ, 503, 325

Anderson J. P. et al., 2014a, MNRAS, 441, 671

Anderson J. P. et al., 2014b, ApJ, 786, 67

Anderson J. P. et al., 2016, A\&A, 589, A110

Anderson J. P. et al., 2018, Nature Astron., 2, 574

Appenzeller I. et al., 1998, The Messenger, 94, 1

Arcavi I. et al., 2010, ApJ, 721, 777

Arcavi I. et al., 2011, ApJ, 742, L18

Arcavi I. et al., 2012, ApJ, 756, L30

Arcavi I. et al., 2017, ApJ, 837, L2

Bacon R. et al., 2014, The Messenger, 157, 13

Barbon R., Ciatti F., Rosino L., 1979, A\&A, 72, 287

Barbon R., Benetti S., Cappellaro E., Patat F., Turatto M., Iijima T., 1995, A\&AS, 110, 513

Baron E. et al., 1995, ApJ, 441, 170

Bayless A. J., Even W., Frey L. H., Fryer C. L., Roming P. W. A., Young P. A., 2015, ApJ, 805, 98

Benvenuto O. G., Bersten M. C., Nomoto K., 2013, ApJ, 762, 74

Bersten M. C. et al., 2012, ApJ, 757, 31

Bersten M. C. et al., 2018, Nature, 554, 497

Black C. S., Milisavljevic D., Margutti R., Fesen R. A., Patnaude D., Parker S., 2017, ApJ, 848, 5

Blondin S., Tonry J. L., 2007, ApJ, 666, 1024

Boquien M., Burgarella D., Roehlly Y., Buat V., Ciesla L., Corre D., Inoue A. K., Salas H., 2019, A\&A, 622, A103

Bostroem K. A. et al., 2019, MNRAS, 562

Brimacombe J. et al., 2017, Astron. Telegram., 11074, 1

Brown T. M. et al., 2013, PASP, 125, 1031

Bruzual G., Charlot S., 2003, MNRAS, 344, 1000

Bufano F. et al., 2014, MNRAS, 439, 1807

Buzzoni B. et al., 1984, The Messenger, 38, 9

Chabrier G., 2003, PASP, 115, 763

Chevalier R. A., Fransson C., 1985, in Bartel N., ed., Supernovae as Distance Indicators. Springer, Berlin, p. 123

Chevalier R. A., Fransson C., 1994, ApJ, 420, 268

Chevalier R. A., Soderberg A. M., 2010, ApJ, 711, L40

Chomiuk L., Povich M. S., 2011, AJ, 142, 197

Chonis T. S., Gaskell C. M., 2008, AJ, 135, 264

Chun S.-H., Yoon S.-C., Jung M.-K., Kim D. U., Kim J., 2018, ApJ, 853, 79

Claeys J. S. W., de Mink S. E., Pols O. R., Eldridge J. J., Baes M., 2011, A\&A, 528, A131
Clocchiatti A., Wheeler J. C., 1997, ApJ, 491, 375

de Jaeger T. et al., 2018, MNRAS, 478, 3776

Dessart L., Waldman R., Livne E., Hillier D. J., Blondin S., 2013, MNRAS, 428,3227

Dessart L. et al., 2014, MNRAS, 440, 1856

Elias-Rosa N. et al., 2010, ApJ, 714, L254

Elmhamdi A., Danziger I. J., Cappellaro E., Della Valle M., Gouiffes C., Phillips M. M., Turatto M., 2004, A\&A, 426, 963

Ergon M. et al., 2014, A\&A, 562, A17

Ergon M. et al., 2015, A\&A, 580, A142

Fang Q., Maeda K., 2018, ApJ, 864, 47

Faran T. et al., 2014, MNRAS, 445, 554

Fesen R. A. et al., 1999, AJ, 117, 725

Filippenko A. V., 1988, AJ, 96, 1941

Filippenko A. V., 1997, ARA\&A, 35, 309

Filippenko A. V., Matheson T., Ho L. C., 1993, ApJ, 415, L103

Firth R. E. et al., 2015, MNRAS, 446, 3895

Flewelling H. A. et al., 2016, preprint (arXiv:1612.05243)

Folatelli G. et al., 2014, ApJ, 792, 7

Fransson C., Chevalier R. A., 1989, ApJ, 343, 323

Freudling W., Romaniello M., Bramich D. M., Ballester P., Forchi V., GarcíaDabló C. E., Moehler S., Neeser M. J., 2013, A\&A, 559, A96

Gal-Yam A., 2017, in Alsabti A. W., Murdin P., eds, Handbook of Supernovae. Springer International Publishing, Cham, p. 195

Galbany L. et al., 2016a, AJ, 151, 33

Galbany L. et al., 2016b, MNRAS, 455, 4087

Galbany L. et al., 2018, ApJ, 855, 107

Gangopadhyay A. et al., 2018, MNRAS, 476, 3611

Goldman S. R. et al., 2017, MNRAS, 465, 403

González-Gaitán S. et al., 2015, MNRAS, 451, 2212

Greiner J. et al., 2008, PASP, 120, 405

Gutiérrez C. P. et al., 2014, ApJ, 786, L15

Gutiérrez C. P. et al., 2017a, ApJ, 850, 89

Gutiérrez C. P. et al., 2017b, ApJ, 850, 90

Gutiérrez C. P. et al., 2018, MNRAS, 479, 3232

Hachinger S., Mazzali P. A., Taubenberger S., Hillebrand t W., Nomoto K., Sauer D. N., 2012, MNRAS, 422, 70

Harutyunyan A. H. et al., 2008, A\&A, 488, 383

Heger A., Fryer C. L., Woosley S. E., Langer N., Hartmann D. H., 2003, ApJ, 591, 288

Henden A. A., Levine S., Terrell D., Welch D. L., 2015, American Astronomical Society Meeting Abstracts \#225. p. 336.16

Houck J. C., Fransson C., 1996, ApJ, 456, 811

Huang F. et al., 2015, ApJ, 807, 59

Inserra C. et al., 2011, MNRAS, 417, 261

Inserra C. et al., 2013, A\&A, 555, A142

Jeffery D. J., Branch D., 1990, in Wheeler J. C., Piran T., Weinberg S., eds, Supernovae, Jerusalem Winter School for Theoretical Physics. World Scientific, Singapore, p. 149

Jerkstrand A., 2017, in Alsabti A. W., Murdin P., eds, Handbook of Supernovae. Springer International Publishing, Cham, p. 795

Jerkstrand A., Fransson C., Maguire K., Smartt S., Ergon M., Spyromilio J., 2012, A\&A, 546, A28

Jerkstrand A., Smartt S. J., Fraser M., Fransson C., Sollerman J., Taddia F., Kotak R., 2014, MNRAS, 439, 3694

Jerkstrand A. et al., 2015a, MNRAS, 448, 2482

Jerkstrand A., Ergon M., Smartt S. J., Fransson C., Sollerman J., Taubenberger S., Bersten M., Spyromilio J., 2015b, A\&A, 573, A12 (J15b)

Jerkstrand A. et al., 2020, MNRAS, 494, 2471

Kennicutt R. C., Evans N. J., 2012, ARA\&A, 50, 531

Kochanek C. S. et al., 2017, PASP, 129, 104502

Krühler T. et al., 2008, ApJ, 685, 376

Kumar B. et al., 2013, MNRAS, 431, 308

Kuncarayakti H. et al., 2015, A\&A, 579, A95

Li H., McCray R., 1992, ApJ, 387, 309

Li H., McCray R., 1993, ApJ, 405, 730

Li W. et al., 2011, MNRAS, 412, 1441

Limongi M., Chieffi A., 2018, ApJS, 237, 13 
Maeda K., Nakamura T., Nomoto K., Mazzali P. A., Patat F., Hachisu I., 2002, ApJ, 565, 405

Maeda K., Nomoto K., Mazzali P. A., Deng J., 2006, ApJ, 640, 854

Maeda K. et al., 2008, Science, 319, 1220

Maeda K. et al., 2015, ApJ, 807, 35

Maguire K. et al., 2010, MNRAS, 404, 981

Maguire K. et al., 2012, MNRAS, 420, 3451

Marino R. A. et al., 2013, A\&A, 559, A114

Matheson T. et al., 2000, AJ, 120, 1487

Maund J. R., Smartt S. J., 2009, Science, 324, 486

Maund J. R., Smartt S. J., Kudritzki R. P., Podsiadlowski P., Gilmore G. F., 2004, Nature, 427, 129

Maund J. R. et al., 2011, ApJ, 739, L37

Maund J. R., Fraser M., Reilly E., Ergon M., Mattila S., 2015, MNRAS, 447, 3207

Maurer I., Mazzali P. A., Taubenberger S., Hachinger S., 2010, MNRAS, 409, 1441

Mazzali P. A., Nomoto K., Patat F., Maeda K., 2001, ApJ, 559, 1047

Mazzali P. A. et al., 2005, Science, 308, 1284

Milisavljevic D., Fesen R. A., Gerardy C. L., Kirshner R. P., Challis P., 2010, ApJ, 709, 1343

Minkowski R., 1941, PASP, 53, 224

Modjaz M., Kirshner R. P., Blondin S., Challis P., Matheson T., 2008, ApJ, 687, L9

Modjaz M. et al., 2014, AJ, 147, 99

Modjaz M., Gutiérrez C. P., Arcavi I., 2019, Nature Astron., 3, 717

Morales-Garoffolo A. et al., 2014, MNRAS, 445, 1647

Morales-Garoffolo A. et al., 2015, MNRAS, 454, 95

Nakar E., Piro A. L., 2014, ApJ, 788, 193

Nomoto K., Iwamoto K., Suzuki T., Pols O. R., Yamaoka H., Hashimoto M., Hoflich P., van den Heuvel E. P. J., 1996, in van Paradijs J., van den Heuvel E. P. J., Kuulkers E., eds, IAU Symp. Vol. 165, Compact Stars in Binaries. p. 119

Oke J. B. et al., 1995, PASP, 107, 375

Pastorello A. et al., 2008, MNRAS, 389, 955

Patat F., Chugai N., Mazzali P. A., 1995, A\&A, 299, 715

Perley D. A., 2019, PASP, 131, 084503

Pessi P. J. et al., 2019, MNRAS, 488, 4239

Podsiadlowski P., Joss P. C., Hsu J. J. L., 1992, ApJ, 391, 246

Puls J., Vink J. S., Najarro F., 2008, A\&AR, 16, 209

Richmond M. W., Treffers R. R., Filippenko A. V., Paik Y., Leibundgut B., Schulman E., Cox C. V., 1994, AJ, 107, 1022

Rubin A. et al., 2016, ApJ, 820, 33

Sahu D. K., Anupama G. C., Srividya S., Muneer S., 2006, MNRAS, 372, 1315

Sahu D. K., Anupama G. C., Chakradhari N. K., 2013, MNRAS, 433, 2

Sanders N. E. et al., 2015, ApJ, 799, 208

Schlafly E. F., Finkbeiner D. P., 2011, ApJ, 737, 103

Schlegel E. M., 1996, AJ, 111, 1660

Shappee B. J. et al., 2014, ApJ, 788, 48

Shivvers I. et al., 2017, PASP, 129, 054201

Silverman J. M. et al., 2017, MNRAS, 467, 369

Smartt S. J., 2015, PASA, 32, e016

Smartt S. J., Maund J. R., Hendry M. A., Tout C. A., Gilmore G. F., Mattila S., Benn C. R., 2004, Science, 303, 499

Smartt S. J., Eldridge J. J., Crockett R. M., Maund J. R., 2009, MNRAS, 395, 1409

Smartt S. J. et al., 2015, A\&A, 579, A40

Smith K. W. et al., 2020, PASP, 132, 22

Spyromilio J., 1994, MNRAS, 266, L61

Spyromilio J., Pinto P. A., 1991, in Danziger I. J., Kjär K., eds, Proceedings to the ESO/EIPC Workshop, Supernova 1987A and Other Supernova. ESO, Garching, p. 423

Sravan N., Marchant P., Kalogera V., 2019, ApJ, 885, 130

Szalai T. et al., 2016, MNRAS, 460, 1500

Taddia F. et al., 2016, A\&A, 587, L7

Taggart K., Perley D., 2019, preprint (arXiv:1911.09112)
Taubenberger S. et al., 2009, MNRAS, 397, 677

Taubenberger S. et al., 2011, MNRAS, 413, 2140

Terreran G. et al., 2016, MNRAS, 462, 137

Terreran G. et al., 2019, ApJ, 883, 147

Tomasella L. et al., 2014, Astron. Nachr., 335, 841

Tonry J. L. et al., 2018, PASP, 130, 064505

Tully R. B. et al., 2013, AJ, 146, 86

Uomoto A., 1986, ApJ, 310, L35

Utrobin V. P., Chugai N. N., 2009, A\&A, 506, 829

Valenti S. et al., 2008, MNRAS, 383, 1485

Valenti S. et al., 2014, MNRAS, 438, L101

Valenti S. et al., 2015, MNRAS, 448, 2608

Valenti S. et al., 2016, MNRAS, 459, 3939

Van Dyk S. D., Garnavich P. M., Filippenko A. V., Höflich P., Kirshner R. P., Kurucz R. L., Challis P., 2002, PASP, 114, 1322

Van Dyk S. D., Li W., Filippenko A. V., 2003, PASP, 115, 1289

Van Dyk S. D. et al., 2011, ApJ, 741, L28

van Loon J. T., Cioni M. R. L., Zijlstra A. A., Loup C., 2005, A\&A, 438, 273

Wongwathanarat A., Janka H. T., Müller E., 2013, A\&A, 552, A126

Wongwathanarat A., Müller E., Janka H. T., 2015, A\&A, 577, A48

Woosley S. E., Heger A., 2015, in Vink J. S., ed., Very Massive Stars in the Local Universe. Springer International Publishing, Switzerland, p. 199

Woosley S. E., Weaver T. A., 1995, ApJS, 101, 181

Woosley S. E., Hartmann D., Pinto P. A., 1989, ApJ, 346, 395

Woosley S. E., Eastman R. G., Weaver T. A., Pinto P. A., 1994, ApJ, 429, 300

Woosley S. E., Heger A., Weaver T. A., 2002, Rev. Mod. Phys., 74, 1015

Yaron O., Gal-Yam A., 2012, PASP, 124, 668

Yuan F. et al., 2016, MNRAS, 461, 2003

\section{SUPPORTING INFORMATION}

Supplementary data are available at MNRAS online.

Table A1. ATLAS photometry.

Table A2. Optical photometry from Las Cumbres Observatory.

Table A3. Optical photometry from Asiago, Mount Ekar.

Table A4. Optical photometry from All-Sky Automated Survey for Supernovae.

Table A5. Optical photometry from the Post Observatory SRO.

Table A6. Optical and near-infrared photometry from the GammaRay Burst Optical/Near-Infrared Detector (GROND).

Table A7. Optical photometry from the Multi Unit Spectroscopic Explorer (MUSE).

Table A8. Spectroscopic observations of SN 2017ivv.

Table A9. FWHM velocity and velocity offset for the $\mathrm{H} \alpha, \mathrm{O}$ I, [Fe II], and [Ca II] lines.

Figure B1. Example of single Gaussian fits for [O I] $\lambda \lambda 6300,6364$ (left-hand panels), $\mathrm{H} \alpha$ (central panels), and [Ca II] $\lambda \lambda 7291,7324$ (right-hand panels) at 158, 242, 331, and $520 \mathrm{~d}$ from explosion. On the top of the $[\mathrm{O}$ I] $] \lambda \lambda 6300,6364$ emission profile, the separation of the two peaks is marked in magenta.

Please note: Oxford University Press is not responsible for the content or functionality of any supporting materials supplied by the authors. Any queries (other than missing material) should be directed to the corresponding author for the article.

\section{APPENDIX}

Appendixes are available as supplementary online data. 
${ }^{1}$ Department of Physics and Astronomy, University of Southampton, Southampton SO17 1BJ, UK

${ }^{2}$ INAF - Osservatorio Astronomico di Padova, Vicolo dell'Osservatorio 5, I-35122 Padova, Italy

${ }^{3}$ The Oskar Klein Centre, Department of Astronomy, Stockholm University, AlbaNova, SE-10691 Stockholm, Sweden

${ }^{4}$ Max-Planck-Institute for Astrophysics, Karl-Schwarzschild Strasse 1, D-85748 Garching, Germany

${ }^{5}$ Departamento de Física Teórica y del Cosmos, Universidad de Granada, E-18071 Granada, Spain

${ }^{6}$ European Southern Observatory, Alonso de Córdova 3107, Casilla 19, Santiago, Chile

${ }^{7}$ Max-Planck-Institut für Extraterrestrische Physik, Giessenbachstraße 1, D-85748 Garching, Germany

${ }^{8}$ Tuorla Observatory, Department of Physics and Astronomy, University of Turku, FI-20014 Turku, Finland

${ }^{9}$ Finnish Centre for Astronomy with ESO (FINCA), FI-20014 University of Turku, Finland

${ }^{10}$ CENTRA, Instituto Superior Técnico, Universidade de Lisboa, Av. Rovisco Pais 1, 1049-001 Lisboa, Portugal

${ }^{11}$ School of Physics \& Astronomy, Cardiff University, Queens Buildings, The Parade, Cardiff CF243AA, UK

${ }^{12}$ School of Physics, O'Brien Centre for Science North, University College Dublin, Belfield Dublin 4, Dublin, Ireland

${ }^{13}$ School of Physics, Trinity College Dublin, The University of Dublin, Dublin 2, Ireland

${ }^{14}$ Astrophysics Research Centre, School of Mathematics and Physics, Queens University Belfast, Belfast BT7 1NN, UK

${ }^{15}$ School of Physics and Astronomy, Tel Aviv University, Tel Aviv 69978, Israel

${ }^{16}$ CIFAR Azrieli Global Scholars program, CIFAR, Toronto, Canada

${ }^{17}$ INAF Osservatorio Astronomico di Padova, Vicolo dell'Osservatorio 5, I-35122 Padova, Italy

${ }^{18}$ Astrophysics Research Institute, Liverpool John Moores University, 146 Brownlow Hill, Liverpool L3 5RF, UK

${ }^{19}$ Department of Astronomy, The Ohio State University, $140 \mathrm{~W}$. 18th Avenue, Columbus, OH 43210, USA

${ }^{20}$ Center for Cosmology and AstroParticle Physics (CCAPP), The Ohio State University, 191 W. Woodruff Avenue, Columbus, OH 43210, USA
${ }^{21}$ Department of Physics, University of California,1 Shields Avenue, Davis, CA 95616-5270, USA

${ }^{22}$ Las Cumbres Observatory, Goleta, CA 93117, USA

${ }^{23}$ Department of Physics, University of California, Santa Barbara, CA 93106 , USA

${ }^{24}$ Kavli Institute for Astronomy and Astrophysics, Peking University, $\mathrm{Yi} \mathrm{He}$ Yuan Road 5, Hai Dian District, Beijing 100871, China

${ }^{25}$ Department of Astronomy, School of Physics, Peking University, Yi He Yuan Road 5, Hai Dian District, Beijing 100871, China

${ }^{26}$ The Oskar Klein Centre, Department of Astronomy, Stockholm University, AlbaNova, SE-10691 Stockholm, Sweden

${ }^{27}$ International Center for Relativistic Astrophysics, Piazzale della Repubblica 2, I-65122 Pescara, Italy

${ }^{28}$ Capodimonte Astronomical Observatory, INAF-Napoli, Salita Moiariello 16, I-80131 Napoli, Italy

${ }^{29}$ Department of Particle Physics and Astrophysics, Weizmann Institute of Science, Rehovot 76100 , Israel

${ }^{30}$ Astronomical Observatory, University of Warsaw, Al. Ujazdowskie 4, PL-00-478 Warszawa, Poland

${ }^{31}$ The Observatories of the Carnegie Institution for Science, 813 Santa Barbara St., Pasadena, CA 91101, USA

${ }^{32}$ Center for Astrophysics | Harvard \& Smithsonian, 60 Garden Str, Cambridge, MA 02138, USA

${ }^{33}$ Birmingham Institute for Gravitational Wave Astronomy and School of Physics and Astronomy, University of Birmingham, Birmingham B15 2TT, UK

${ }^{34}$ Institute for Astronomy, University of Edinburgh, Royal Observatory, Blackford Hill EH9 3HJ, UK

${ }^{35}$ Departamento de Ciencias Fisicas, Universidad Andres Bello, Avda. Republica 252, Santiago, Chile

${ }^{36}$ Millennium Institute of Astrophysics (MAS), Nuncio Monseñor Sotero Sanz 100, Providencia, Santiago, Chile

${ }^{37}$ Núcleo de Astronomía de la Facultad de Ingeniería y Ciencias, Universidad Diego Portales, Av. Ejército 441 Santiago, Chile

${ }^{38}$ Institute for Astronomy, University of Hawai'i, 2680 Woodlawn Drive, Honolulu, HI 96822, USA

This paper has been typeset from a $\mathrm{T}_{\mathrm{E}} \mathrm{X} / \mathrm{L} \mathrm{T}_{\mathrm{E}} \mathrm{X}$ file prepared by the author. 